\title{
Functional Characterization of Deformation Fields
}

\author{
ETIENNE CORMAN and MAKS OVSJANIKOV \\ LIX, École Polytechnique, CNRS
}

In this paper we present a novel representation for deformation fields of 3D shapes, by considering the induced changes in the underlying metric. In particular, our approach allows to represent a deformation field in a coordinate-free way as a linear operator acting on real-valued functions defined on the shape. Such a representation both provides a way to relate deformation fields to other classical functional operators and enables analysis and processing of deformation fields using standard linear-algebraic tools. This opens the door to a wide variety of applications such as explicitly adding extrinsic information into the computation of functional maps, intrinsic shape symmetrization, joint deformation design through precise control of metric distortion, and coordinate-free deformation transfer without requiring pointwise correspondences. Our method is applicable to both surface and volumetric shape representations and we guarantee the equivalence between the operator-based and standard deformation field representation under mild genericity conditions in the discrete setting. We demonstrate the utility of our approach by comparing it with existing techniques and show how our representation provides a powerful toolbox for a wide variety of challenging problems.

Categories and Subject Descriptors: I.3.5 [Computer Graphics]: Computational Geometry and Object Modeling_Geometric algorithms, languages, and systems

General Terms: Algorithms, Design

Additional Key Words and Phrases: Shape exploration, functional maps

ACM Reference Format:

\section{INTRODUCTION}

Designing and analyzing shape deformations is a central problem in computer graphics and geometry processing, with applications in scenarios such as shape manipulation [Yu et al. 2004; Sorkine and Alexa 2007], animation and deformation transfer [Sumner and Popović 2004], shape interpolation [Kilian et al. 2007; VonTycowicz et al. 2015], and even anisotropic meshing [Panozzo et al. 2014] among myriad others. Traditionally, shape deformation has

Permission to make digital or hard copies of part or all of this work for personal or classroom use is granted without fee provided that copies are not made or distributed for profit or commercial advantage and that copies show this notice on the first page or initial screen of a display along with the full citation. Copyrights for components of this work owned by others than ACM must be honored. Abstracting with credit is permitted. To copy otherwise, to republish, to post on servers, to redistribute to lists, or to use any component of this work in other works requires prior specific permission and/or a fee. Permissions may be requested from Publications Dept., ACM, Inc., 2 Penn Plaza, Suite 701, New York, NY 10121-0701 USA, fax +1 (212) 869-0481, or permissions@acm.org.

(c) YYYY ACM 0730-0301/YYYY/17-ARTXXX \$10.00

DOI 10.1145/XXXXXXX.YYYYYYY

http://doi.acm.org/10.1145/XXXXXXX.YYYYYYY
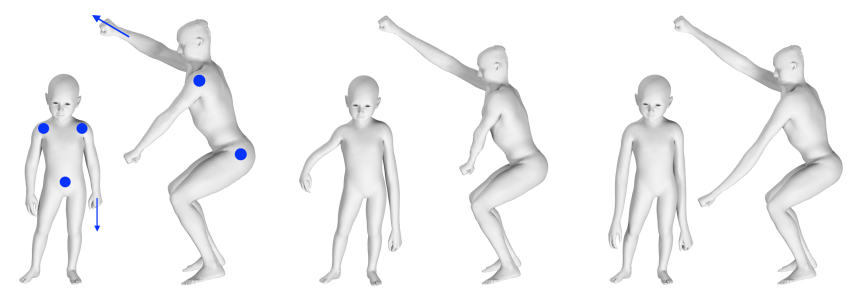

Fig. 1: An example of joint deformation design using our framework where all objectives are easily expressed as linear constraints. Left pair: A set of local constraints for the deformation fields on two different shapes. Middle pair: Joint deformation design by a direct transfer of the coordinate functions of the deformation field. This method yields undesirable effects as it is rotation dependent. Right pair: Joint deformation design with a soft (functional) map and no pointwise correspondences between the shapes.

been motivated by interactive applications in which the main goal is to design a deformation that satisfies some user-prescribed handle constraints while preserving the main structural properties of the shape. In other applications, such as shape interpolation and deformation transfer, that lack handle constraints, the goal is to design a global deformation field that would satisfy some structural properties as well as possible.

In both types of applications, most approaches are based on specifying a deformation energy and providing a method to optimize it. On the other hand, several works have demonstrated that by choosing an appropriate representation for shape deformations, many tasks can become significantly easier, and in particular can help to enforce certain properties of the deformation field, which are otherwise very difficult to access and optimize for. In addition to the classical per-vertex displacement vectors, such representations have included gradient-based deformations [Yu et al. 2004; Zayer et al. 2005], Laplacian-based approaches [Lipman et al. 2004; Sorkine et al. 2004] and Möbius transformations in the context of conformal deformations [Crane et al. 2011; Vaxman et al. 2015] among others.

At the same time, a number of recent works have shown that many basic operations in geometry processing can be viewed as linear operators acting on real-valued functions defined on the shapes. This includes the functional representation of mappings or correspondences acting through composition [Ovsjanikov et al. 2012; Pokrass et al. 2013], representations of vector fields as derivations [Pavlov et al. 2011; Azencot et al. 2013] and formulation of shape distortion via shape difference operators [Rustamov et al. 2013]. One advantage of these representations is that linear operators can be naturally composed, which makes it easy to define, for example, the push-forward of a vector field with respect to a mapping, if both are represented as linear operators, or to solve for Killing vector fields, by composition between a derivation and the Laplacian operator. Moreover, by using a consistent functional representation these techniques often alleviate the need for point-wise correspondences, which can be difficult to obtain, as shown very recently for example in a work on joint cross-field design [Azencot et al. 2017].

While tangent vector fields are classically understood as operators (derivations) in differential geometry, extrinsic vector fields 
do not enjoy a similar property. Our main goal is to provide a coordinate-free representation of extrinsic vector fields (that we also call deformation fields) as functional operators, which will prove useful for analysis and design of shape deformations. As we demonstrate below our representation greatly simplifies certain tasks such as intrinsic symmetrization, the computation of mappings by composition with other operators, and joint deformation design without requiring point-wise mappings. Moreover, it provides an explicit link between deformation fields and the changes in intrinsic metric quantities, which can be useful in a variety of analysis and deformation processing tasks.

For example, consider two shapes shown in Fig. 1 (left). By using our framework, it is possible to combine local deformation constraints with intrinsic objectives such as constructing a deformation field that is as-isometric-as-possible. Moreover, our representation allows to relate deformations on multiple shapes in a coordinate-free way, enabling deformation transfer and joint design using only soft, functional correspondences as shown in Fig. 1 (right).

\section{RELATED WORK}

Shape deformation is one of the oldest and best-researched topics in computer graphics and geometry processing. We therefore only mention works most directly related to ours and refer the interested reader to surveys including [Nealen et al. 2006; Botsch and Sorkine 2008] and [Botsch et al. 2010] (Chapter 9).

A multitude of methods exists for surface deformation starting with the seminal work of [Terzopoulos et al. 1987], its early followups including [Celniker and Gossard 1991; Welch and Witkin 1992] and the multi-scale variants, such as [Zorin et al. 1997; Kobbelt et al. 1998; Guskov et al. 1999] among many others. Similarly to our approach, many of these techniques are based on optimizing the so-called elastic thin shell energy that measures stretching and bending, and which is often linearized for efficiency. In the majority of cases, deformations are represented explicitly as extrinsic vector fields defined on a surface, making deformation transfer difficult in the absence of precise pointwise correspondences.

A number of methods have proposed alternative representations for deformation fields, which greatly simplify certain tasks in design and analysis. This includes gradient-based techniques [Yu et al. 2004; Zayer et al. 2005] which consider the deformation field by aligning its gradient with a set of local per-triangle transformations. By working in gradient space, constraints can be posed independently on the triangles and then optimized globally by solving the Poisson equation. Similarly, Laplacian-based techniques [Sorkine et al. 2004; Lipman et al. 2004; Nealen et al. 2005] are based on defining shape deformations by manipulating per-vertex differential coordinates (Laplacians) in order to match some target Laplacian coordinates. Such differential coordinates enable direct editing of local shape properties, which can be especially beneficial for preserving and manipulating the high-frequency details of the surface. However, these coordinates are typically not rotationally invariant and additional steps are necessary to introduce invariance [Sorkine et al. 2004; Lipman et al. 2004; Paries et al. 2007].

More recently, a number of methods have introduced representations for mesh deformations specifically geared towards particular shape manipulations, such as computing conformal transformations by designing special maps into the space of quaternions [Crane et al. 2011] or by using face-based compatible Möbius transformations [Vaxman et al. 2015]. These techniques are rotationally invariant and coordinate-free, while being restricted to special types of manipulations. Another technique, closely related to ours, designs shape deformations by constructing a continuous divergence-free vector field [von Funck et al. 2006], and applying path line integration to obtain a deformed shape. We also consider the effect of the deformation on the metric, but both analyze the distortion of arbitrary extrinsic vector fields and show how they can be represented in coordinate-free way as linear functional operators.

Our use of spectral techniques and functional maps for representing deformation fields is also related to previous works in spectral shape processing, including the early approaches of Lévy and colleagues and their extensions [Lévy 2006; Vallet and Lévy 2008; Dey et al. 2012] and more recent techniques such those based on coupled quasi-harmonic bases and functional maps [Kovnatsky et al. 2013; Yin et al. 2015]. In these and related methods deformation fields are represented as triplets of functions, which encode displacement in each spatial coordinate. Although this representation is simple and naturally fits with the functional map framework, it suffers from several drawbacks. First, it is not rotationally invariant and induces artefacts if the shapes are not pre-aligned or are in different poses (see e.g., Figure 1). Perhaps more fundamentally, such a representation is not "shape-aware" since it does not reflect the change in the (e.g., metric) structure of the shapes induced by the deformation, which reduces its utility in deformation analysis and design. We demonstrate through extensive experiments, that by using our coordinate-free representation we can avoid these limitations and open the door to entirely novel design and analysis applications, such as intrinsic symmetrization (Section 8.2), which cannot be achieved using previous methods.

Our approach of considering the deformation via its induced metric distortion is also related to the work of [Eigensatz and Pauly 2009] and [Sela et al. 2015] who manipulate shapes by explicitly editing their curvature properties. Moreover, our use of the strain tensor in characterizing metric distortion is closely related to the applications in various physically based deformation scenarios including [Thomaszewski et al. 2009; Müller et al. 2014] among many others (see also the surveys on physically based elastic deformable models [Nealen et al. 2006; Rumpf and Wardetzky 2014]). Our approach is also related to the works that aim to design as-isometricas-possible shape deformations [Zhang et al. 2015; Solomon et al. 2011; Martinez Esturo et al. 2013]. Similarly to the latter work, our framework is general and allows an arbitrary prescribed distortion, although our method works directly on surface representations and moreover enables applications such as joint deformation design.

Finally, our framework for joint design is related to the deformation transfer and interpolation techniques such as [Sumner and Popović 2004; Baran et al. 2009] and [Kilian et al. 2007] to name a few. Our approach is different in that we place special emphasis on relating deformations between shapes with only soft (or functional) correspondences, which are often much easier to obtain than detailed point matches. Moroever, rather than transporting Jacobian matrices associated with the deformation, which requires both a pre-alignment and an approximate triangle-to-triangle map (as done in [Sumner and Popović 2004]) we study and transport the change in the intrinsic metric structure directly. As we show below, this results in better joint deformation design especially given approximate functional maps, and shapes in arbitrary poses.

Thus, in contrast to the majority of existing techniques our goal is to devise a coordinate-free representation of extrinsic deformations as linear functional operators, by making an explicit connection between the extrinsic deformations and the change in intrinsic metric quantities. As such, our representation fits within the recent line of work that represents many operations in geometry processing as functional operators, including mappings or correspondences [Ovsjanikov et al. 2012; Pokrass et al. 2013], representations of vector fields as derivations [Pavlov et al. 2011; Azencot et al. 2013] and 
the formulation of shape distortion via shape difference operators [Rustamov et al. 2013]. Therefore, although we build on classical constructions such as the infinitesimal strain tensor, we show how they can be exploited to create a functional representation of shape deformation, which can be used in conjunction with other operators. As we demonstrate below, our representation is particularly useful for analysing and manipulating the effect of the deformation on the shape structure and for relating deformations across shapes, with only soft correspondences between them. In particular, it enables applications such as intrinsic symmetrization, joint deformation design and allows to introduce extrinsic information in the computation of functional maps. Remarkably, we prove that together with the classical Laplace-Beltrami operator, our approach leads to a complete (up to rigid motion) coordinate-free functional shape representation, which opens the door to new shape processing applications.

\section{OVERVIEW}

The rest of the paper is organized as follows: first, we define the functional deformation field representation using the classical notions of the Levi-Civita connection and the strain tensor, and list the main properties of this representation (Section 4). We then provide a link between this definition and the previously proposed shape difference operators, by considering their infinitesimal extensions, introducing a new unified operator, and proving the equivalence between the two definitions (Section 5). In Sections 6 and 7 we provide a discretization of all of these notions, and show that they preserve the main properties of the continous counterparts. Finally, we illustrate the utility of our representation by describing several novel application scenarios, which range from functional map inference, to intrinsic symmetrization and deformation field design that all exploit the properties of our representation and its relation to other previously proposed linear operators (Section 8). Note that Sections 5 and 7 can be skipped by readers that are not interested in the connection to shape difference operators.

To summarize, our main contributions include:

-Introducing functional deformation fields as a way to represent extrinsic vector fields in a coordinate-free way as operators acting on functions, represented as matrices in the discrete setting.

-Providing a link between functional deformation fields and the previously proposed shape difference oprators, which leads to both a new unified shape difference and alternative functional deformation fields, which can be made sensitive to specific (e.g., non-conformal) classes of distortions.

- Showing how functional deformation can be used to naturally add extrinsic information (second fundamental form) into the computation and analysis of functional maps. We also prove that together with the Laplace-Beltrami operator, they provide a complete coordinate-free shape characterization up to rigid motions.

-Describing how this representation enables a number of novel applications including intrinsic shape symmetrization, deformation design and functional deformation transfer without pointwise correspondences.

\section{EXTRINSIC VECTOR FIELDS AS OPERATORS}

In this section we provide a coordinate-free representation of extrinsic vector fields by considering their action on the underlying shape metric. Throughout this section we assume that we are dealing with a smooth surface $M$ without boundary embedded in $\mathbb{R}^{3}$. The appropriate discretization of all the concepts introduced in this section will be given in Section 6.
The Levi-Civita Covariant Derivative. We first need to introduce some fundamental notions from differential geometry. In particular, we will use the classical Levi-Cevita connection to define derivatives on a surface. More precisely, given a tangent vector $u$ at some point $p \in M$, and an extrinsic vector field $V$ on $M$, consider an arbitrary curve $\gamma(t)$ on $M$ such that $\gamma(0)=p$ and $\gamma^{\prime}(0)=u$. Then, we let $\bar{\nabla}_{u} V=\left.\frac{\partial V(\gamma(t))}{\partial t}\right|_{t=0}$. Here $\bar{\nabla}_{u} V$ is the standard covariant derivative of the ambient space. Note that at a fixed point $p \in M, \bar{\nabla}_{u} V$ is a vector in $\mathbb{R}^{3}$. We can project the covariant derivative onto the tangent plane at $p$ to obtain a vector in the tangent plane, which is denoted simply by $\nabla_{u} V$ where $\nabla$ is the Levi-Cevita connection on $M$ extended naturally to extrinsic vector fields, ([do Carmo 2013] p. 126). We also remark that for any vector $x$ in the tangent space, $\left\langle\nabla_{u} V, x\right\rangle=\left\langle\bar{\nabla}_{u} V, x\right\rangle$, which we will use in our discretization.

The fundamental object that we consider below is the infinitesimal strain tensor, which can be understood as a bilinear form, acting on pairs of vectors $x, y$ in the tangent plane of a point $p \in M$. Namely, given an extrinsic vector field $V$, the infinitesimal strain tensor $\mathcal{L}_{V} \mathbf{g}(x, y)$ is defined as:

$$
\mathcal{L}_{V} \mathbf{g}(x, y)=\left\langle x, \nabla_{y} V\right\rangle+\left\langle\nabla_{x} V, y\right\rangle
$$

This quantity has the advantage of being linear in the vector field $V$, which makes it easy to handle for deformation and vector field design and therefore has been used in a wide variety of works in computer graphics [Nealen et al. 2006].

Physically, this tensor represents the infinitesimal stretch that the object undergoes at each point. Thus, the eigenvector associated to the largest eigenvalue of $\mathcal{L}_{V} \mathbf{g}$ (which can be thought of simply as a symmetric $2 \times 2$ matrix) at a point $p$, corresponds to the tangent vector $x$ that represents the local direction of maximal stretch.

With these definitions in hand we propose to consider a linear functional operator $E^{V}$, which we will use to capture and manipulate a deformation field $V$. Both the input and the output of our operator are smooth real-valued functions defined on the surface. This operator is defined implicitly, in the same spirit as the shape difference operators introduced by Rustamov et al. [Rustamov et al. 2013] as follows: for every pair of real-valued functions $f, g$ we require:

$$
\int_{M}\left\langle\nabla g, \nabla E^{V}(f)\right\rangle \mathrm{d} \mu=\int_{M} \mathcal{L}_{V} \mathbf{g}(\nabla g, \nabla f) \mathrm{d} \mu .
$$

The following proposition guarantees that $E^{V}$ is well-defined.

PROPOSITION 1. For any extrinsic vector field $V$ there is a unique linear functional operator $E^{V}$ that satisfies Eq. (2) above. Moreover, this operator is linear in both the vector field $V$ and function $f$.

In the rest of the paper we call the linear functional operator $E^{V}$, a functional deformation field representation of $V$. Our main goal is to design, manipulate and analyze extrinsic vector fields $V$ through their associated linear functional operators $E^{V}$. This approach has already proved useful in the context of manipulating maps or correspondences [Ovsjanikov et al. 2012], tangent vector fields [Azencot et al. 2013] and shape distortions [Rustamov et al. 2013]. In particular, these works have helped to establish a general formalism of shape manipulation through the associated linear functional operators, which can "communicate" by composition. This allows, for example, to transfer tangent vector fields across shapes without assuming pointwise correspondences [Azencot et al. 2013] or to design very efficient shape matching algorithms using the functional 

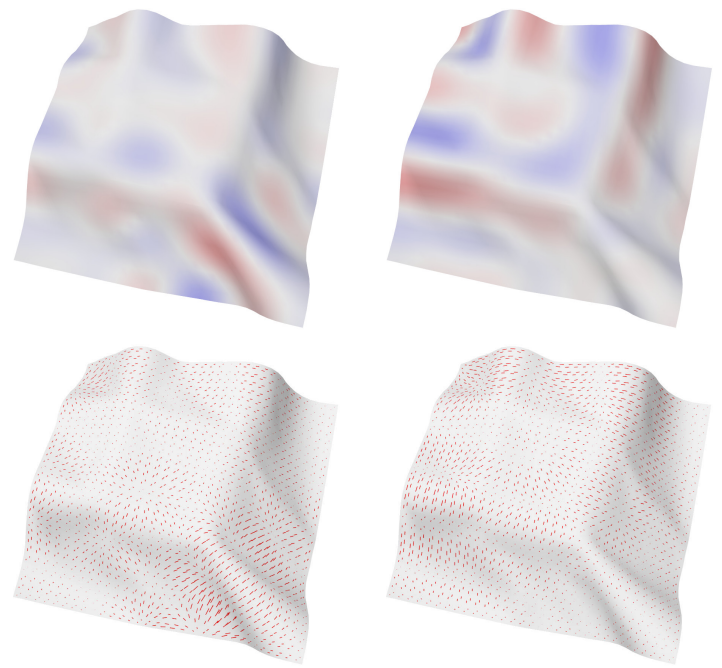

1st eigenfunction

2nd eigenfunction

Fig. 2: Two eigenfunctions associated with the largest eigenvalues of the operator infinitesimal shape difference $E^{n}$ for the normal field $n$. The gradients of these functions represent the direction of maximal curvature (Bottom row).

map representation [Ovsjanikov et al. 2016]. Therefore, inspired by these works, we propose to extend this framework to also include extrinsic (or deformation) fields. As we show below, our representation naturally fits within the general functional operator formalism and enables a number of novel applications.

\subsection{Key Properties of Functional Deformation Fields}

Second-fundamental form representation. One interesting special case to consider is the interpretation of $E^{V}$ when the deformation field is the normal field $V=n$. By using Eq. (1) it is possible to see ([do Carmo 2013] p.128) that the covariant derivative of the normal yields the second fundamental form denoted by $\mathbf{h}_{p}: T_{p} M \times T_{p} M \rightarrow \mathbb{R}$, more precisely $\mathcal{L}_{n} \mathbf{g}=-2 \mathbf{h}$. Therefore the operator $E^{n}$ captures the action of curvature on functions, since:

$$
\int_{M}\left\langle\nabla f, \nabla E^{n}(g)\right\rangle \mathrm{d} \mu=-2 \int_{M} \mathbf{h}(\nabla f, \nabla g) \mathrm{d} \mu .
$$

From a theoretical point of view the knowledge of the LaplaceBeltrami operator gives access to the first fundamental form and $E^{n}$ yields information about the second. Thus these two operators jointly provide a coordinate-free representation of the embedding.

The operator $E^{n}$ can be used to obtain a multi-scale representation of curvature information on the triangle mesh, as shown in Figure 2. In particular, the eigenfunctions corresponding to the largest eigenvalues of $E^{n}$, are those that align the best with the maximal principal curvature direction, and can be obtained even if $E^{n}$ is represented in a reduced functional basis, making the computation less sensitive to noise in the triangulation. Moreover, as we demonstrate in Section 8.1, the operator $E^{n}$ can be used to inject extrinsic information into the computation of functional maps.

Composition with mappings. In many applications we are interested in the relation between deformations on multiple surfaces related by a mapping. In particular given a deformation field $U_{M}$ of shape $M$ and a diffeomorphism $\varphi: M \rightarrow N$ with the associated functional map (pullback) $C_{\varphi}$ of functions from $N$ to $M$, one can define a deformation field $V_{N}$ of shape $N$ that produces the same metric distortion. Instead of looking directly at the deformation of the metric, which might require a mapping between individual triangles [Sumner and Popović 2004], we account for the action of the metric on functions:

$$
E^{U_{M}} C_{\varphi}(f)=C_{\varphi} E^{V_{N}}(f) \forall f \in C^{\infty}(N)
$$

In other words, $V_{N}$ can be obtained by considering an extrinsic vector field, whose operator representation has the same effect on functions when composed with the functional map $C_{\varphi}$ as $E^{U_{M}}$. This property allows us to relate deformation fields without requiring point-to-point correspondences between shapes, by simply considering the commutativity of the operators $C_{\varphi}$ and $E$. We illustrate this in Figure 5 and use it in Section 8.4 for deformation transfer and deformation symmetrization on meshes with different connectivities with only a functional map known between them. Furthermore, this approach is applicable to design deformations jointly on two shapes, such that they are consistent with the functional map $C_{\varphi}$ and even as a regularizer in map computation.

Vector field representation. In general the operator $E^{V}$ does not uniquely define an extrinsic vector field. From Def. 2 it can be shown that the kernel of $V \mapsto E^{V}$ coincides with the vector fields satisfying $\mathcal{L}_{V} \mathbf{g}=0$. In case of a volumetric manifold (i.e. $M \subset \mathbb{R}^{3}$ ) the kernel of our operator is restricted to infinitesimal rigid motions (see Theorem 1.7-3 in [Ciarlet 2000]) and thus provides a complete representation of extrinsic vector fields. In the case of a surface embedded in $\mathbb{R}^{3}$ the kernel of $E^{V}$ includes infinitesimal isometries such as Killing vector fields but also local normal fields in planar areas. No rigidity result seems to be known for smooth surfaces. However, as we demonstrate below, in the discrete case of shapes represented as triangle meshes, it can be shown that for almost all surfaces the kernel of $V \mapsto E^{V}$ consists only of rigid deformations (Prop. 4). Note that we place no restriction on the magnitude of the deformation fields. Thus, although our construction is based on the infinitesimal strain tensor, the extrinsic vector fields themselves are not limited to infinitesimal (or local) deformations. Finally, as we show below, our constructions can be easily extended to the case of tetrahedral meshes, resulting in a complete operator-based representation for deformation fields of volumes, not sensitive to the exceptional cases, present in the case of surfaces.

\section{RELATION TO SHAPE DIFFERENCE OPERATORS}

The functional deformation field representation introduced above is closely related to the previously proposed shape difference operators. In this section we describe this relation in detail, and highlight the following two key insights: 1) How our analysis leads to a novel unified shape difference operator, and 2) How alternative functional deformation field representations can be constructed, to be sensitive to only a particular class of metric distortions. Our analysis also sheds light on the discretization of functional deformation fields. Nevertheless, the discussion in this section is not required for the understanding of either the implementation or the results of our approach, apart from the intrinsic symmetrization application (Sec. 8.2), in which we use this relation. As such, this section can be skipped by readers not interested in these relations.

\subsection{Shape Difference Operators}

Introduced by [Rustamov et al. 2013], the shape difference operators describe a shape deformation by considering the change of inner 
products between functions. Namely, given a pair of shapes $M, N$ and a diffeomorphism $\varphi: N \rightarrow M$, with the associated linear functional map (pullback) defined by $C_{\varphi}(f)=f \circ \varphi$, the authors introduce the area-based and conformal shape difference operators $D_{A}$ and $D_{C}$ respectively, as linear operators acting on (and producing) real-valued functions on $M$ implicitly via the following equations:

$$
\begin{aligned}
& \left\langle f, D_{A}(g)\right\rangle_{L^{2}(M)}:=\left\langle C_{\varphi}(f), C_{\varphi}(g)\right\rangle_{L^{2}(N)} \quad \forall f, g \\
& \left\langle f, D_{C}(g)\right\rangle_{H_{0}^{1}(M)}:=\left\langle C_{\varphi}(f), C_{\varphi}(g)\right\rangle_{H_{0}^{1}(N)} \quad \forall f, g
\end{aligned}
$$

where the inner products are defined as $\langle f, g\rangle_{L^{2}(M)}:=\int_{M} f g \mathrm{~d} \mu$ and $\langle f, g\rangle_{H_{0}^{1}(M)}:=\int_{M}\langle\nabla f, \nabla g\rangle \mathrm{d} \mu$.

The existence and the linearity of the operators $D_{A}$ and $D_{C}$ is guaranteed by the Riesz representation theorem. As shown in [Rustamov et al. 2013], for smooth surfaces, the map $\varphi$ is areapreserving (resp. conformal) if and only if $D_{A}$ (resp. $D_{C}$ ) is the identity map between functions. From this it follows that $\varphi$ is an isometry if and only if $D_{A}$ and $D_{C}$ are both identity.

Note that in the discrete setting the shape difference operators are obtained simply by considering transposes and inverses of the functional map and Laplacian matrices, as highlighted in [Rustamov et al. 2013]. This makes properties such as existence and linearity trivial to see. Below we adopt the continuous (surface) formulation proposed in the original article as it helps to highlight both the generality of these concepts and also the relation to our representation of extrinsic vector fields.

Infinitesimal Shape Difference Operators. Our main goal in this section is to consider a one-parameter family of shapes $M_{t}$, given by displacing the points of a base shape along some fixed deformation field. Specifically, given a surface $M$ embedded in $\mathbb{R}^{3}$ we consider a family $M_{t}$, parameterized by a scalar $t$ and given by $p_{t}=p_{0}+t V\left(p_{0}\right)$, where $p_{0}$ is a fixed point in $\mathbb{R}^{3}$, and $V(p)$ is a vector in $\mathbb{R}^{3}$ that represents the displacement of the point.

Now consider the family of maps $\varphi_{t}: M \rightarrow M_{t}$, given trivially via $\varphi_{t}(p)=p_{t}$, and the associated functional maps $C_{\varphi_{t}^{-1}}$ mapping functions from $M_{0}$ to $M_{t}$. This gives rise to a one-parameter family of shape difference operators $D_{t}^{V}$ (which can be taken either to be the area or conformal-based operators). We then introduce the infinitesimal shape difference operator as follows:

DEFINITION 1. The infinitesimal area-based shape difference operator associated with an extrinsic vector field $V$ on a surface $M$ is defined as:

$$
E_{A}^{V}:=\left.\frac{\partial D_{A t}^{V}}{\partial t}\right|_{t=0},
$$

We define the infinitesimal conformal shape difference operator $E_{C}^{V}$ similarly by replacing $D_{A}^{V}$ by $D_{C}^{V}$ on the right side of Eq. (5).

Remark that since both $E_{A}^{V}$ and $E_{C}^{V}$ are defined as derivatives of a one-parameter family of linear operators acting on real-valued functions on a surface, both the range and the domain of these operators are also real-valued functions on $M$. Moreover, as $D_{A}^{V}$ and $D_{C}^{V}$ reflect (or, equivalently, are sensitive to) changes in the area and conformal metric structure, this implies that $E_{A}^{V}$ and $E_{C}^{V}$ will only reflect extrinsic vector fields up to infinitesimally areapreserving or conformal deformations. This naturally raises the question of whether there exists another "unified" shape difference operator $D_{I}$, which would be sensitive to general (non-isometric) metric changes. If so, would such $D_{I}$ lead to an infinitesimal shape difference $E_{I}$ that would agree with the definition of $E^{V}$ given in Eq. (2)? Below, we provide precisely such a definition which both
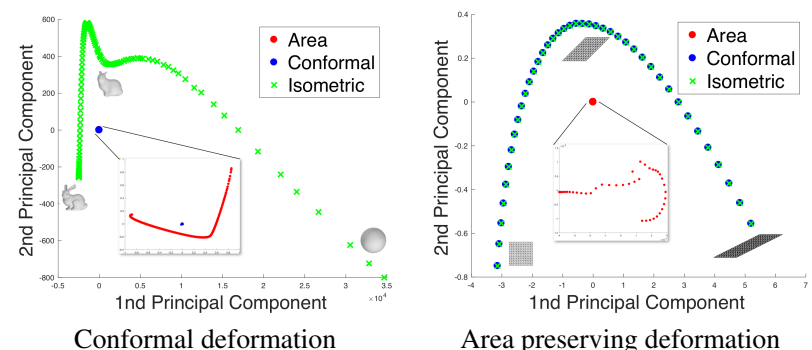

Area preserving deformation

Fig. 3: Left: Approximately conformal deformation of a bunny into a sphere. The PCA applied to shape differences confirms the presence of large area and isometric distortion in contrast to small conformal distortion. Right: Area preserving deformation of a plane. The area-based shape difference is almost constant while conformal and isometric differences agree.

extends the applicability of shape difference operators and helps to establish a deeper link with our functional deformation fields.

Unified shape difference. The main reason for which $D_{C}$ is only sensitive to conformal changes is that both the inner product and the integration are taken on the target shape. To define a unified shape difference taking into account all intrinsic changes one should compare the pullback metric to the metric on $M$ while keeping the integrating measure fixed. We thus propose a unified shape difference operator $D_{I}$ that fully characterizes isometric distortion.

Definition 2. Assuming that $\varphi: N \rightarrow M$ is a diffeomorphism, the unified shape difference $D_{I}: \mathcal{C}^{\infty}(M) \rightarrow \mathcal{C}^{\infty}(M)$ is defined implicitly by:

$$
\left\langle f, D_{I}(g)\right\rangle_{H_{0}^{1}(M)}:=\int_{M} C_{\varphi^{-1}}\left(\left\langle\nabla C_{\varphi}(f), \nabla C_{\varphi}(g)\right\rangle\right) \mathrm{d} \mu^{M} .
$$

The existence of $D_{I}$ is once again guaranteed by the Riesz representation theorem. Moreover, as we claimed above, the following proposition (proved in the supplemental material) shows that the unified shape difference fully characterizes isometric deformation.

Proposition 2. $D_{I}(f)=f$ for all $f \in \mathcal{C}^{\infty}(M)$ if and only if $\varphi$ is an isometry.

To illustrate the properties of the three shape differences we use a simple low-dimensional description of a shape collection in Figure 3. Here we choose a fixed base shape and compute the shape difference matrices with respect to the remaining shapes in a collection. Then, we represent each shape by its shape difference matrix and plot them as points in PCA space. Figure 3 represents the conformal deformation of a bunny into a sphere as viewed by the three shape differences. As expected $D_{C}$ is almost identity while the area and isometric shape differences both capture the distortion. In the second experiment, shown in Figure 3, we explore another collection obtained by the shearing of a plane patch. As this deformation is area preserving, the area-based shape difference provides no information, unlike the other two operators.

With the definition of the unified shape difference $D_{I}$ in hand, we introduce its infinitesimal counterpart $E_{I}$ by following the same construction as done in (5) above. The following proposition (proved in the supplemental material) characterizes these new operators.

Proposition 3. Let $V$ be a smooth deformation field on $M$, the derivatives of $D_{A}, D_{C}$ and $D_{I}$ at time zero satisfy for all smooth 
functions $f, g$ :

$$
\begin{aligned}
& \left\langle f, E_{A}^{V}(g)\right\rangle_{L^{2}(M)}=\int_{M} \operatorname{div}(V) f g \mathrm{~d} \mu, \\
& \left\langle f, E_{C}^{V}(g)\right\rangle_{H_{0}^{1}(M)}=\int_{M} \operatorname{div}(V)\langle\nabla f, \nabla g\rangle-\mathcal{L}_{V} \mathbf{g}(\nabla f, \nabla g) \mathrm{d} \mu, \\
& \left\langle f, E_{I}^{V}(g)\right\rangle_{H_{0}^{1}(M)}=-\int_{M} \mathcal{L}_{V} \mathbf{g}(\nabla f, \nabla g) \mathrm{d} \mu .
\end{aligned}
$$

As can be seen, the infinitesimal shape differences inherit the properties of the original operators. Namely, $E_{A}^{V}$ vanishes if and only if $\operatorname{div}(V)$ is equal to zero, i.e., whenever $V$ infinitesimally preserves the volume form. On the conformal side, finding an extrinsic vector field $V$ such that $E_{C}^{V}=0$ is equivalent to solving the conformal Killing equation: $\mathcal{L}_{V} \mathbf{g}=\operatorname{div}(V) \mathbf{g}$ characteristic of a conformal vector field. Both properties combined lead to an isometric deformation induced by the vector field $V$ captured by $E_{I}$.

Moreover Prop. 3 reveals a clear link between shape differences:

$$
\left\langle f, E_{I}^{V}(g)\right\rangle_{H_{0}^{1}}=\left\langle f, E_{C}^{V}(g)\right\rangle_{H_{0}^{1}}-\left\langle 1, E_{A}^{V}(\langle\nabla f, \nabla g\rangle)\right\rangle_{L^{2}} .
$$

Thus, intuitively, the operator $E_{I}$, representing isometric distortion, can be decomposed into an area and a conformal part. We note that linear dependence between shape operators shown in Eq. (6) can be understood as the decomposition of the matrix $\mathcal{L}_{V} \mathbf{g}$ into a trace free part, linked to the conformal Killing equation, and a divergence part, related to the change in area.

Finally, this proposition shows that the functional deformation field representation introduced in Section 4 is exactly the same as the infinitesimal shape difference operator $E_{I}^{V}$ arising from the unified shape difference. Remarkably, this relation also holds exactly in the discrete setting as we show in Section 7.

Summary. To summarize, in this section we first showed that an alternative way for constructing a linear functional operator representation of extrinsic vector fields consists in considering a family of deformations of the shape, constructing the associated shape difference operators, and taking their derivative at zero, which leads to infinitesimal shape differences. This also suggests alternative functional deformation field operators, sensitive only to specific kinds of deformations (e.g., non area-preserving or non-conformal). Finally, we showed that by modifying the definition of shape differences, a new, unified difference operator can be constructed and that its derivative at time zero leads precisely to the functional deformation field formulation introduced in the previous section.

\section{DISCRETE SETTING}

In this section we provide the discretization of functional deformation fields. For this, we first propose a particular discretization of the Levi-Civita connection and the Lie derivative of the metric on the triangle mesh, which leads to a simple formula for the operator $E^{V}$. In the following section, Sec. 7, which can be skipped similarly to Sec. 5, we demonstrate that the deep connection between functional deformation fields and infinitesimal shape difference operators also holds in the discrete setting.

Throughout this section, we assume that we are given a manifold triangle mesh. We denote by $(\mathcal{X}, \mathcal{E}, \mathcal{F})$ respectively the set of vertices, edges and faces. We will consider the deformation field $V$, which we also call an extrinsic vector field, to be given as a three-dimensional vector per vertex.
Discrete connection. To build the discrete operator $E^{V}$ we need a consistent discretization of the Levi-Civita connection. While several discrete connections have been proposed (e.g. [Azencot et al. 2015; Liu et al. 2016]), because of the special nature of our problem, we choose to build our own. This is because, applications such as parallel transport require that the vectors $u, v$ and $\nabla_{u} v$ are expressed in the same space (at vertex or face or edge) so often an averaging step has to be introduced to transfer, for example, a facebased representation of a vector to an edge based representation. In our setting such a requirement is not needed and it is easier to distinguish tangent vector fields that will be expressed by one vector per face and extrinsic vector fields expressed at vertices. Thus, our goal is to obtain a connection of the ambient space $\bar{\nabla}_{u} V$ where $u$ is a tangent vector and $V$ is an extrinsic vector field :

$$
\begin{aligned}
\bar{\nabla}: \mathbb{R}^{3|\mathcal{F}|} \times \mathbb{R}^{3|\mathcal{V}|} & \rightarrow \mathbb{R}^{3|\mathcal{F}|} \\
(u, V) & \mapsto \bar{\nabla}_{u} V
\end{aligned}
$$

We build the connection $\bar{\nabla}$ using finite differences as follows. Since extrinsic vector fields are defined at vertices the differences are taken along the edges.

DEFINITION 3. In a given triangle $T \in \mathcal{F}$ the ambient covariant derivative along the edge $e_{i j}$ is defined by

$$
\left(\bar{\nabla} \underset{\frac{e_{i j}}{\left\|e_{i j}\right\|}}{ } V\right)_{T}=\frac{V_{i}-V_{j}}{\left\|e_{i j}\right\|} .
$$

Thus the ambient connection in the directions $E=\left(e_{i j}, e_{j k}\right)$ can be stored in a matrix

$$
\left(\bar{\nabla}_{E} V\right)_{T}=\left(V_{i}-V_{j} V_{j}-V_{k}\right) .
$$

Then, given any tangent vector $x=E \alpha$, the covariant derivative in its direction can be computed as $\bar{\nabla}_{x} V=\left(\bar{\nabla}_{E} V\right) \alpha$.

Given the expression above, the discrete Lie derivative of the metric at triangle $T$ follows immediately, using Eq. (1). Namely for any pair of tangent vectors $x=E \alpha, y=E \beta$ in the triangle $T$, we have:

$$
\mathcal{L}_{V} \mathbf{g}(x, y)_{T}=\left\langle x,\left(\bar{\nabla}_{E} V\right) \beta\right\rangle+\left\langle\left(\bar{\nabla}_{E} V\right) \alpha, y\right\rangle .
$$

If $W_{M}$ denotes the cotangent-weight Laplacian, which classically represents the inner products of $H_{0}^{1}$ (and is also called stiffness matrix), we obtain the discrete functional deformation field operator from its definition (2):

$$
f^{\top} W_{M} E^{V} g=-\sum_{T \in \mathcal{F}} \mathcal{L}_{V} \mathbf{g}(\nabla f, \nabla g)_{T} \mu(T) .
$$

Then we obtain $E^{V}(u)=W_{M}^{-1} H$, where $H$ is a Laplacian matrix whose weights depend on the extrinsic vector field:

$$
\begin{aligned}
& (H)_{i j}=\frac{1}{2} \sum_{j \sim i}\left(c\left(T_{\alpha_{i j}}\right)+c\left(T_{\beta_{i j}}\right)\right), \\
& c(T)=\left(\left\langle e_{j k}, V_{j}-V_{i}\right\rangle+\left\langle e_{i j}, V_{j}-V_{k}\right\rangle\right) \frac{1}{4 \mu(T)} \\
& -\operatorname{Tr}\left(\left(E^{\top} E\right)^{-1} E^{\top}\left(\nabla_{E} V\right)\right) \frac{\left\langle e_{j k}, e_{k i}\right\rangle}{\mu(T)} .
\end{aligned}
$$

The computations can be found in the supplemental material.

\subsection{Properties}

Interestingly, many of the properties of the continuous operators are satisfied exactly by their discrete counterparts. 
Linearity. The discretization $E^{V}(f)$ naturally preserves the linearity with respect to both $V$ and $f$ which is very convenient for practical purposes.

In practice, it is often convenient to use a functional basis, so that any function can be represented as a linear combination of some basis functions $\phi_{i}$. Given such a basis, the operator $E^{V}$ can be seen as the (possibly infinite) matrix: $E^{V}{ }_{i j}=\left\langle\phi_{i}, E^{V}\left(\phi_{j}\right)\right\rangle_{L^{2}(M)}$. The choice of basis depends on the application. Since we are interested in smooth deformations of a surface, we take a subset of the smoothest functions given by the first $k$ eigenfunctions of the Laplace-Beltrami operator. In that case, $E^{V}$ will be represented simply as a $k \times k$ matrix. As shown in Figure 13 the size of the basis $k$ affects the deformation field that we can represent and recover. Increasing $k$ allows a more faithful representation of high frequency deformation fields.

The linearity with respect to $V$ allows the same operation for vector fields. Therefore, if the deformation field is given in some basis $V=\sum_{i} \alpha_{i} X_{i}$ then the operator reads $E^{V}=\sum_{i} \alpha_{i} E^{X_{i}}$ This means that when designing a deformation field $V$ we can consider an objective as a function of the coefficients $\alpha$.

Vector Fields representation. In the continuous setting the kernel of $V \mapsto E^{V}$ is the set of infinitesimal isometries. However, to the best of our knowledge, there is no characterization of how often this set is reduced to rigid motion. In the particular setting of our discretization some standard results can be applied, however.

PROPOSITION 4. For almost all triangle meshes $M$ without boundary, the operator $E^{V}$ uniquely defines the extrinsic vector field $V$ up to rigid motion.

Thanks to this proposition, we can guarantee that $E^{V}$ is almost always a complete coordinate-free representation of extrinsic vector fields $V$. Triangle meshes containing perfectly flat neighborhoods fall in the category of shapes on which the map $V \mapsto E^{V}$ is not injective. Namely, since by definition of the strain tensor (Eq. 2), whenever $\nabla_{x} V$ and is normal to the surface for all $x$, (as is the case when e.g. $V$ is a normal field on a flat part and zero elsewhere), the tensor $\mathcal{L}_{V}$ will lead to the zero operator. Although we have found that for organic and natural shapes, such vector fields are rare or non-existent, they can nevertheless be important for coarse meshes or man-made objects with flat areas.

\subsection{Construction for Tetrahedral Meshes}

To remedy this problem, we extend our discretization to tetrahedral meshes thus avoiding ill-defined vector fields as the kernel of $V \mapsto$ $E^{V}$ is of dimension 6 (translations and infinitesimal rotations). For this we follow the construction provided in Section 6, by adapting it to tet meshes. Namely, we extend the ambient covariant derivative matrix $E$ in Eq. (7) to three dimensions, by considering the covariant derivative along three directions of a tet mesh, and thus storing a $3 \times 3$ matrix $\bar{\nabla}_{E} V$ per simplex. We then use Eq. (8) without any modifications to obtain a discretization of the functional deformation fields on tet meshes. The final resulting formula for the matrix $E_{I}^{V}$ is provided in the supplementary material.

We compare the stability of our representation between tetrahedral and triangle meshes in Figure 4, by plotting the condition number of the linear system for recovering the vector field $V$ from its operator representation $E_{I}^{V}$ in the case of surface (triangle) and tet mesh reprentations of a cube. We note that although the condition number becomes unbounded for the triangle mesh representation as the shape approaches a flat cube, it nevertheless remains remarkably stable: even at 0.9 where the sphere is almost a cube the condition

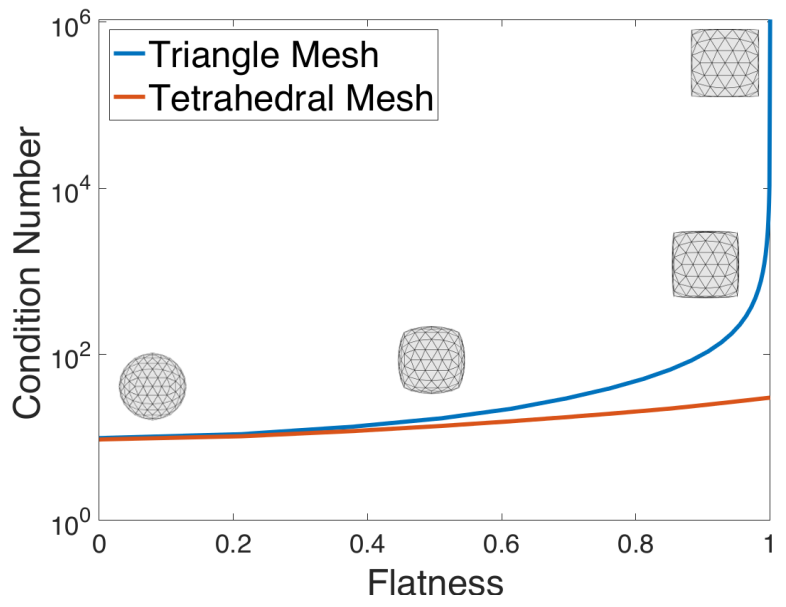

Fig. 4: Comparison of the condition number of the linear map $V \mapsto E^{V}$ when computed on triangle and tetrahedral meshes with respectively 162 and 163 vertices. The condition number is computed as the ratio of the largest and the 7th lowest singular value to avoid infinitesimal rotations and translations naturally mapped to zero. We consider the collection of meshes formed by a sphere morphing into a cube. For a triangle mesh the condition number goes to infinity as the shape becomes increasingly flatter. When considering a tetrahedral mesh the condition number remains bounded.

number is about 100 . In contrast the condition number for tet meshes remains bounded even for a perfectly flat shape.

An important consequence of Proposition 4 is that deformation fields are fully encoded by the operator $E^{V}$ up to infinitesimal rigid motions. Therefore any deformation can be recovered regardless of its scale and nature. For instance Figure 13 shows that noninfinitesimal global rotations are correctly encoded and recovered from our operator representations.

\section{DISCRETE INFINITESIMAL SHAPE DIFFERENCES}

Similarly to the link between established in Section 5 between our initial definition for functional deformation fields and infinitesimal shape differences, we can consider an alternative discretization to the one above by considering a family of deformed meshes and taking the derivative of shape difference operators. In this section we show that this approach leads to exactly the same result, which means that remarkably Proposition 3 is satisfied exactly in the discrete setting. To demonstrate this result we first provide a discretization of the unified shape difference operator and then highlight the link between the infinitesimal shape difference operators and functional deformation fields. Similarly to Section 5, this section is primarily of conceptual interest and can be skipped by readers who wish to proceed to the practical results.

To compute the shape differences we start from the discretization of the inner product $\langle., .\rangle_{H_{0}^{1}}$ using standard first order finite elements. We will denote by $L$ the classical cotangent Laplacian matrix, $W$ the inner product of $H_{0}^{1}$ and $A$ the lumped mass matrix such that $L=A^{-1} W$. As before $\mu$ is a measure and $\mu(T)$ denotes the area of triangle $T$.

\subsection{Discrete unified shape difference}

The discretization of the unified shape difference is straightforward when $N$ and $M$ are triangle meshes and share the same connectivity. 

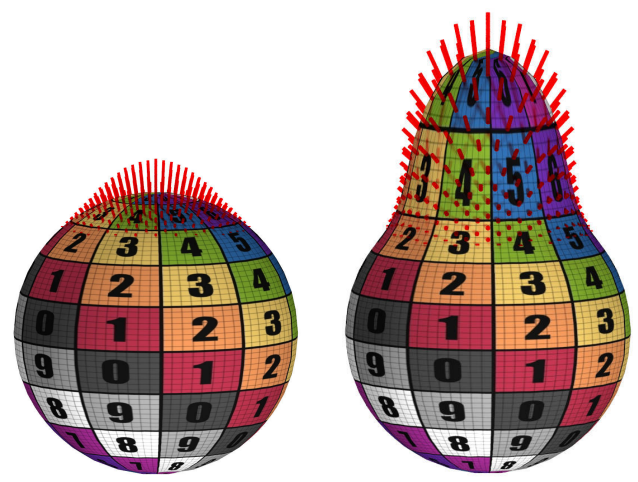

Fig. 5: Example of deformation fields that commute with the diffeomorphism represented by texture transfer. Note that both the direction and the magnitude of the vector field have to adapt to the underlying geometry to produce the same metric distortion.

In Definition 2 given above, the gradients and the point-wise scalar products are taken on $N$ while the measure $\mathrm{d} \mu^{M}$ comes from $M$. Therefore the right hand side can be discretized by a modified cotangent weight formula:

$$
\begin{aligned}
W_{M} D_{I} & =W_{N}^{M}, \text { where } \\
\left(W_{N}^{M}\right)_{i, j} & =\frac{1}{2}\left(\frac{\mu^{M}\left(T_{\alpha}\right)}{\mu^{N}\left(T_{\alpha}\right)} \cot \alpha_{i j}^{N}+\frac{\mu^{M}\left(T_{\beta}\right)}{\mu^{N}\left(T_{\beta}\right)} \cot \beta_{i j}^{N}\right) .
\end{aligned}
$$

Here $T_{\alpha}, T_{\beta}$ are the two triangles adjacent to edge $i, j$, which is opposite to angles $\alpha$ and $\beta$, while $\mu^{M}$ and $\mu^{N}$ are the triangle areas on shapes $M$ and $N$ respectively. Note that $W_{N}^{M}$ differs from the standard cotangent weight matrix $W_{N}$ only by the ratio of weights per triangle. Moreover,notice that if the transformation is area preserving for all triangles then $D_{I}$ reduces to the conformal shape difference defined in [Rustamov et al. 2013] (Option 1 in Section 5).

From the expression above it follows that $D_{I}=W_{M}^{-1} W_{N}^{M}$.

Expression in a basis. Similarly to the construction given in [Rustamov et al. 2013] we can also express the unified shape difference when the basis $\Phi_{M}$ on the source shape $M$ is given by the eigenfunctions of the Laplace-Beltrami operator. In that case, using a diagonal matrix $\Lambda_{M}$ of eigenvalues, $D_{I}$ becomes:

$$
D_{I}=\Lambda_{M}^{-1} \Phi_{M}^{T} W_{N}^{M} \Phi_{M}
$$

This expression has the advantage of avoiding the inverse of a large sparse matrix, and can be used to analyze deformation of a shape with fixed connectivity in multi-scale basis, which can make the computations resilient to local perturbations (see Option 3 in Section 5 of [Rustamov et al. 2013] ).

Approximation with a functional map. Note that both expressions above assume that the source and target meshes share the same connectivity. When the meshes have different connectivity this discretization requires a map between triangles making it challenging to use in practice. To overcome this problem we approximate this discrete formulation by transferring the weights on triangles to lumped weights on vertices. The approximation then reduces to the usual discrete quantities:

$$
\left(\tilde{W_{N}^{M}}\right)_{i j} \approx \frac{\sum_{t \sim i} \mu^{M}\left(T_{t}\right)}{\sum_{t \sim i} \mu^{N}\left(T_{t}\right)} \frac{1}{2} \sum_{j \sim i}\left(\cot \alpha_{i j}^{N}+\cot \beta_{i j}^{N}\right) .
$$

We recognize here the cotangent Laplacian $L_{N}$ with lumped area weights, namely $A_{M} L_{N}$. In the case of meshes with different connectivity, this remark suggests the following approximation of the isometric shape difference, valid only in a discrete sense, for an arbitrary linear functional map $C$ between $M$ and $N$ :

$$
f^{\top} A_{M} L_{M} D_{I} g \approx f^{\top} A_{M} C^{-1} L_{N} C g .
$$

In the reduced basis of the Laplacian eigenvectors, the approximation of the shape difference becomes $D_{I} \approx \Lambda_{M}^{-1} C^{-1} \Lambda_{N} C$, which preserves the principal property of the operator: $D_{I}$ is identity if and only if the deformation is an isometry since the Laplacian on $N$ has to be equal to the Laplacian on $M$. We used this discretization in Figure 3 and observed that the two expressions given above typically produce similar results.

\subsection{Shape difference derivative}

Suppose that each vertex $p_{i}$ of the mesh is displaced by the vector $V_{i}$ by $p_{i}^{t}=p_{i}+t V_{i}$. This produces a family of triangle meshes $\left(\mathcal{X}^{t}, \mathcal{E}, \mathcal{F}\right)$ with identical connectivity. It is now possible to take the derivative with respect to $t$ of Eq. (9) at time 0 . This way we obtain a discretization of the infinitesimal shape differences. Remarkably the resulting discretization is strictly identical to the discrete functional operator $E^{V}$ proposed in Section 6 based on the discrete Levi-Civita connection.

PROPOSITION 5. The discretization of $E$ based on the discrete Levi-Civita connection is equivalent to the one obtained by differentiating the unified shape difference operator.

Shape difference decomposition. Since the discretization using a discrete connection and through the time derivative agree, the decomposition described by Eq. (6) is also satisfied exactly. Namely, the matrix $E^{V}{ }_{C}$ representing the discrete infinitesimal conformal shape difference splits into the discrete functional deformation field $E^{V}$ and an appropriately defined discrete divergence:

$$
f^{\top} W E_{C}^{V} g=f^{\top} W E^{V} g+\sum_{T \in \mathcal{F}} \operatorname{div}(V)_{T}\langle\nabla f, \nabla g\rangle_{T} \mu(T) .
$$

Thus, the decomposition of $E^{V}$, representing isometric distortion, into area and conformal parts given in Eq. (6) in the continuous case holds exactly in the discrete case as well.

\section{EXPERIMENTS}

In this section we apply our constructions to various tasks in shape correspondence, deformation design and analysis. As our framework relies on manipulating and inverting moderately-sized matrices, all of the applications are very efficient, even when combining multiple objectives.

In some applications (Sec. 8.2 - 8.4), it is necessary to recover the deformation field from its function operator representation. For this, we use a reduced basis and recover the coefficients of the deformation field by solving a convex problem similar to basis pursuit. Namely, given a target functional deformation field operator $E^{V}$ represented as a matrix, we solve for $V$ via:

$$
\min _{\alpha}\left\|\sum_{i} \alpha_{i} E^{X_{i}}-E^{V}\right\|_{F}^{2}+\|\alpha\|_{1}
$$

where $\alpha$ is a vector of coefficients and $E^{X_{i}}$ are the functional representation of the $i^{\text {th }}$ deformation field in an overcomplete basis. Of course, the choice of basis is application dependent. The simplest and most general choice would be to consider a basis which consists of independent displacements at each vertex of the given mesh. For a mesh with $n_{V}$ vertices, this would result in $3 n_{V}$ unknowns when 


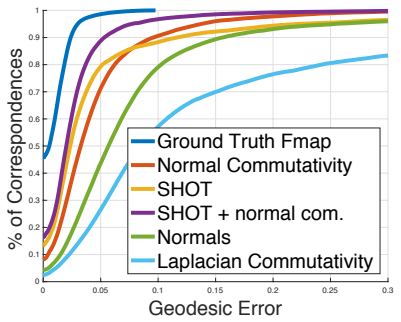

Same pose

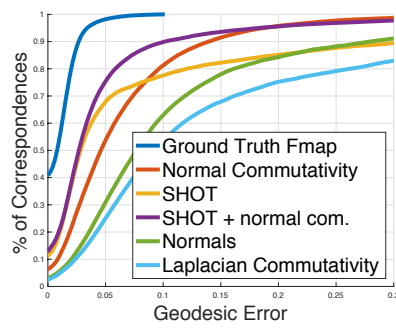

Different Pose
Fig. 6: Percentage of correspondences within a geodesic ball averaged on a 50 pairs for a two shape matching problems: left different character taking the same pose, right different character taking different pose.

solving for a deformation field, which is feasible when $n_{V}$ is small (and was used in the experiment in Figure 13), but can be inefficient for larger meshes. When needed (Sec. 8.2 - 8.4) we use the following deformation bases in the experiments below:

-The simplest option is to take the eigenfunctions of the LaplaceBeltrami operator as the basis for each component of the deformation field. While simple, this basis might not preserve rotation invariance.

-Alternatively we construct a basis via modal analysis of a deformation energy. In particular we consider an energy of the form $V \mapsto \int_{M}\|\vec{\nabla} V\|^{2} \mathrm{~d} \mu$. This corresponds to the energy on a particular discretization of the Bochner Laplacian of extrinsic vector fields. To obtain the basis we take the eigenvectors of the Hessian of the energy, which correspond to smooth deformation fields.

-Lastly, we use the handle-based deformation model described in [Adams et al. 2008]. Unlike the other families, the deformation fields arising from this model are compactly supported and therefore better suited to reproduce local deformations.

\subsection{Functional map inference}

In our first application, we show how our functional deformation field representation can be used as a regularization in shape matching problems. In particular, we show how this representation can be used to add extrinsic information to the computation of functional maps [Ovsjanikov et al. 2012]. The vast majority of the existing methods for shape correspondence with functional maps use the assumption of approximate intrinsic isometries (see [Ovsjanikov et al. 2016] for an overview) and are either purely intrinsic or inject extrinsic or embedding-dependent information by adding extrinsic descriptors. On the other hand, our functional deformation field representation provides a natural coordinate-free way to add embedding-dependent information into the map estimation pipeline. In particular, our approach below is based on the following key observation:

Proposition 6. Given a pair of surfaces $M, N$ embedded in $3 D$, and a diffeomorphism $T: N \rightarrow M$, let $C$ be the corresponding functional map $L^{2}(M) \rightarrow L^{2}(N)$. Then $M$ and $N$ are related by a rigid motion in space if and only if:

$$
\left\|C \Delta_{M}-\Delta_{N} C\right\|+\left\|C E_{M}^{n}-E_{N}^{n} C\right\|=0,
$$

where $\Delta$ are the LB operators, while $E^{n}$ are functional deformation fields arising from the normal fields.

This proposition is simply a consequence of the fundamental theorem of surface theory and the relation between functional deformation fields and the second fundamental form described in
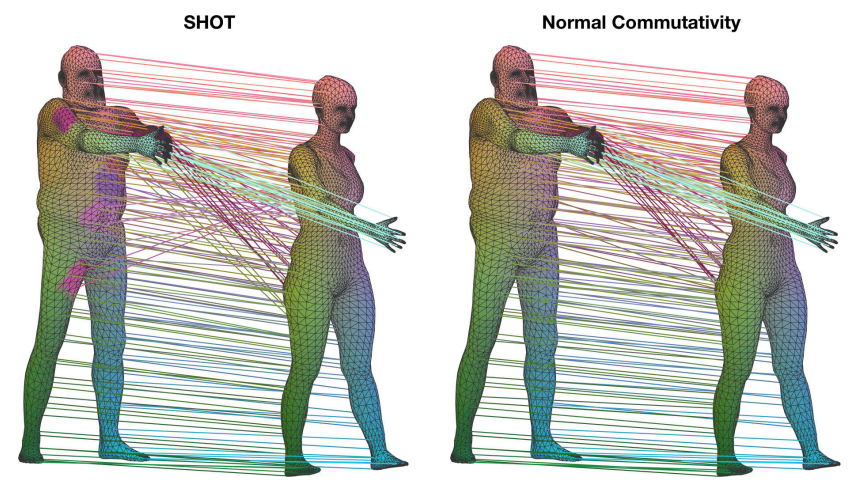

Fig. 7: Representation of the point-to-point map evaluated in Figure 6. The RGB channel (left column) represents the $x y z$-coordinates, which are transferred using the recovered point-to-point map. The correspondences obtained with SHOT (left) are precise near sharp features (e.g., on the fingers) whereas our constraint (right) is informative on the entire shape.

Section 4.1. Note that enforcing the condition of this proposition in practice reduces simply to penalizing the lack of commutativity of the functional map $C$ with predefined operators, which can be done efficiently in practice. Therefore, we can see that functional deformation fields provide an effective way to capture embedding-dependent information in a coordinate-free way that fully characterizes the shape geometry up to rigid motions.

Inspired by this observation, we propose to solve the following problem: given two shapes and a sparse set of correspondences recover a dense map. The shapes come from the Faust dataset [Bogo et al. 2014] and we are given five corresponding landmarks at the hands, feet and head. The baseline method following the logic of the original paper is to represent the landmark points as delta functions $\delta_{M}$ and $\delta_{N}$ and look for the most isometric functional map $C$ : $L^{2}(M) \rightarrow L^{2}(N)$, by enforcing commutativity with the LaplaceBeltrami operator. Thus, the straightforward approach would be to solve the optimization problem:

$$
\min _{C}\left\|C \Delta_{M}-\Delta_{N} C\right\|_{F}^{2} \quad \text { s.t. } \quad C \delta_{M}=\delta_{N} .
$$

The basic way to add extrinsic information to this problem is to constraint the map to preserve extrinsic descriptors noted $F_{M}, F_{N}$ respectively on $M, N$ :

$$
\min _{C}\left\|C \Delta_{M}-\Delta_{N} C\right\|_{F}^{2}+\left\|C F_{M}-F_{N}\right\|_{F}^{2} \quad \text { s.t. } \quad C \delta_{M}=\delta_{N} .
$$

We evaluate two commonly used descriptors:, 1) the normal vector field encoded as three independent functions and 2) the purely extrinsic descriptor SHOT [Tombari et al. 2010] successfully used for solving partial matching problems [Litany et al. 2017].

We compare these descriptor-based approaches to our coordinatefree extrinsic constraint. According to Prop. 6 if a diffeomorphism commutes with the Laplace-Beltrami operator and $E^{n}$ then the shapes admit the same embedding. Our new optimization problem thus reads:

$$
\min _{C}\left\|C \Delta_{M}-\Delta_{N} C\right\|_{F}^{2}+\left\|C E^{n}{ }_{M}-E^{n}{ }_{N} C\right\|_{F}^{2} \text { s.t. } C \delta_{M}=\delta_{N} \text {. }
$$

Once the functional map are obtained they are converted to a point-to-point map using the knn-algorithm as described in [Ovsjanikov et al. 2012]. The results are shown for two non-isometric shape matching problems: different characters taking the same pose and taking different pose. Figure 6 shows the percentage of correspondences within a given geodesic distance. Interestingly, SHOT 


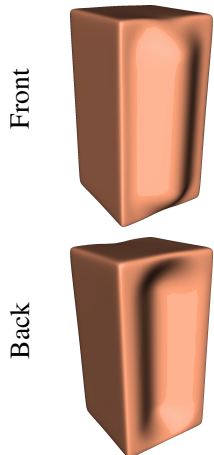

Initial

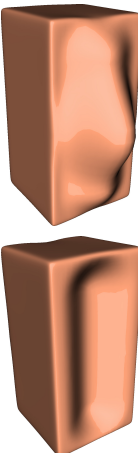

Deformed



Intrinsic Sym

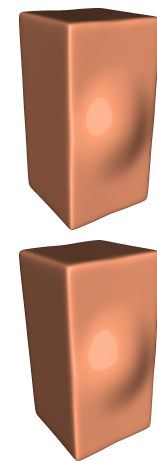

Extrinsic Sym.
Fig. 8: An initially intrinsically symmetric bar (far left) is artificially made asymmetric (middle left). Our algorithm (middle right) is able to recover the symmetry while maintaining the intrinsic structure of the shape. In comparison an extrinsic symmetrization scheme (far right) would erase the intrinsic structure.

provides valuables information on sharp features allowing accurate matching near salient points but tends to fail on featureless regions. In comparison our constraint provides information everywhere on the shape making the results less subject to obvious mismatching but it is less informative on the placement of salient features. This intuition is confirmed by Figure 7 which provides a visualization of the point-to-point correspondences by transferring the coordinates functions encoded as RGB channels. Finally, the combination of those two constraints overcomes the limitations of both methods taken independently.

\subsection{Intrinsic Symmetrization}

In this section we show how our representation of deformation fields can be used to deform shapes to make them more intrinsically symmetric, while keeping their general pose. For example, Figure 8 shows a shape with important features which would be lost by an extrinsic symmetrization scheme. However an intrinsic symmetrization algorithm would preserve those features while recovering the symmetry. This way, our goal is similar to the one of [Zheng et al. 2015] although our approach, unlike theirs, avoids the computation of a skeleton and is purely intrinsic. More precisely, given a base shape $M$ and a self-map $\pi: M \rightarrow M$ we would like to compute the shape $M^{\prime}$ such that the self-map $\psi$ on $M^{\prime}$ is an isometry. If we denote by $\varphi: M^{\prime} \rightarrow M$ the map from $M$ to $M^{\prime}$ then the symmetry map on the deformed shape is given by $\psi=\varphi^{-1} \circ \pi \circ \varphi$. Using Prop. 2 the isometric constraint is satisfied if and only if the unified shape difference $D_{I}^{\psi}$, computed with the map $\psi$, equals identity. If $C_{T}$ is the functional map representation of a map $T$, then after simplification this is equivalent to $D_{I}^{\pi} C_{\pi}^{-1} D_{I}^{\varphi} C_{\pi}=D_{I}^{\varphi}$ (see supplementary material).

Note, however that every intrinsically symmetric shape would be a solution of this equation. Therefore we regularize the problem by imposing that $\varphi$ should be as-isometric-as possible. The equality conditions are enforced in the least squares sense leading to the optimization problem:

$$
\min _{\varphi}\left\|D_{I}^{\pi} C_{\pi}^{-1} D_{I}^{\varphi} C_{\pi}-D_{I}^{\varphi}\right\|_{F}^{2}+\tau\left\|D_{I}^{\varphi}-I\right\|_{F}^{2} .
$$

The optimization (10) is restricted to the set of diffeomorphisms so a direct approach is challenging to use in practice. A more tractable method is to use functional deformation fields as a first
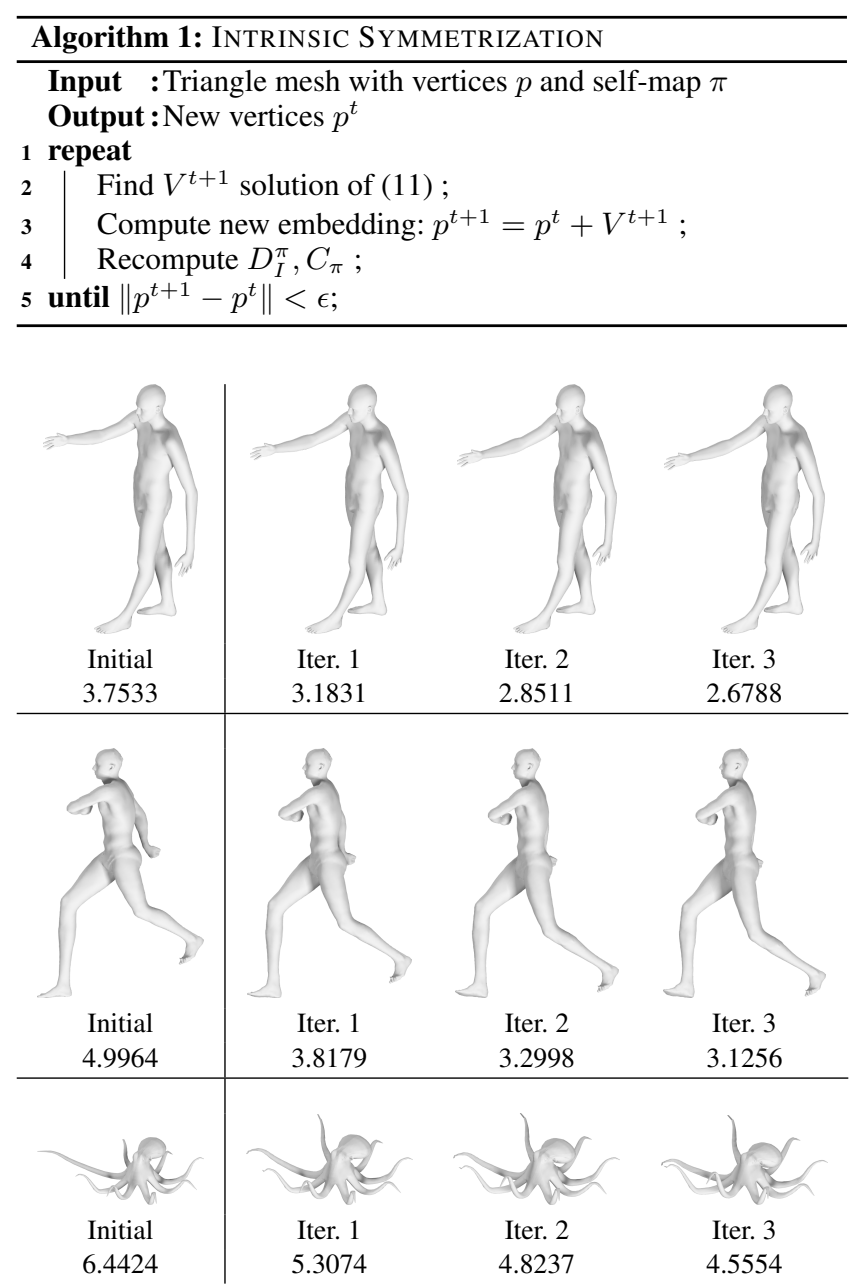

Fig. 9: Three iterations of our intrinsic symmetrization method, Algorithm 1, given an approximate symmetry map. At each step we measure the distance to the symmetry by the Frobenius norm between the intrinsic shape difference induced by the symmetry map and identity, namely $\left\|D_{I}-I\right\|_{F}$. Although not directly taken into account, this energy decreases at each iteration. Note that our algorithm works with any type of symmetries: see bottom row for a non-reflectional symmetry.

order approximation of shape differences and thus find the deformation field that solves (10) to first order. After linearization, Eq. (10) becomes:

$$
\min _{V}\left\|D_{I}^{\pi} C_{\pi}^{-1} E^{V} C_{\pi}-E^{V}-I+D_{I}^{\pi}\right\|_{F}^{2}+\tau\left\|E^{V}\right\|_{F}^{2} .
$$

This linearization suggests an iterative algorithm (described in Algorithm 1) which alternates between solving the linearized problem (11) and computing the new vertex positions. In practice, we construct an over-complete dictionary of deformation fields, composed of the three bases described at the beginning of Section 8 , compute the optimal deformation field by solving for the coefficients $\alpha$.

Figure 9 shows two examples where our method successfully recovers intrinsic symmetry from meshes with outstretched parts. In [Zheng et al. 2015] the authors propose a method based on skeleton driven deformation to achieve intrinsic symmetry but limited to reflectional symmetries. Our method does not require such as- 


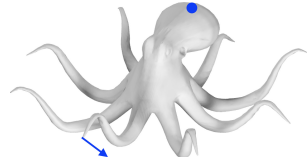

Constraints
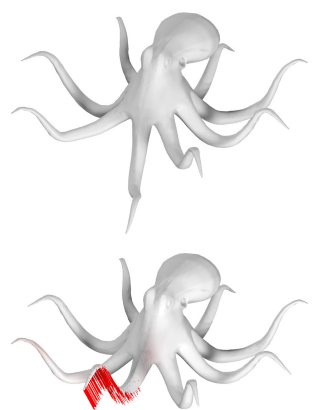

As-isometric-as possible
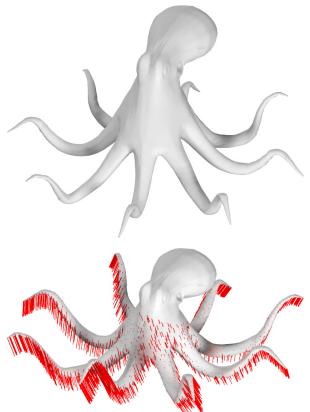

Symmetry
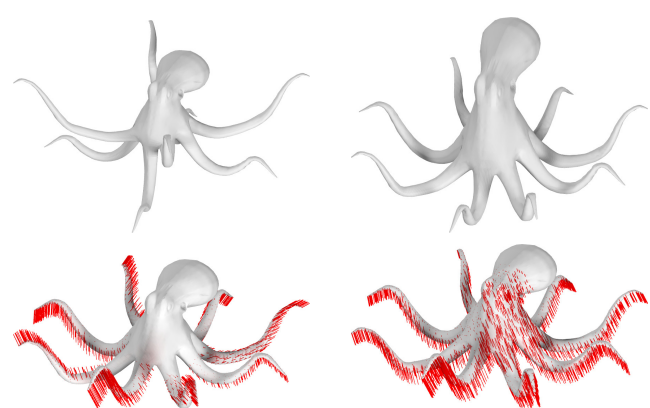

Anti-symmetry

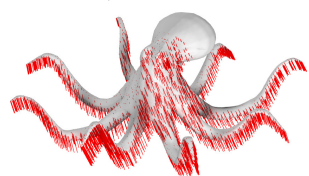

Laplacian Reg.

Fig. 10: We design deformations respecting the directional constraints shown on the far left and minimizing various criteria (from left to right): the infinitesimal shape difference leading to the most isometric vector field, the commutativity with a self-map, the anti-commutativity with the same self-map and the commutativity with the Laplace-Beltrami operator.



Vector field

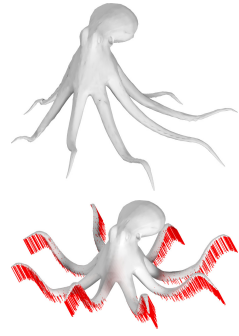

Symmetry

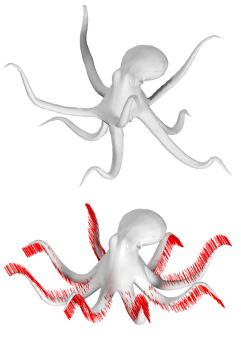

Anti-symmetry
Fig. 11: We design deformations by projecting the vector field shown on the far left onto the space of symmetric and anti-symmetric functions. This direct approach does not deliver the expected results found in Figure 10.

sumptions and works for any given self-mapping (e.g. bottom row in Figure 9). Note also that our deformation field representation is essential in this scenario, since for example, representing deformation fields through displacement functions would not provide information on the necessary (or induced) metric distortion.

\subsection{Deformation design}

Since our operator is linear with respect to the deformation field one can easily combine multiple constraints to the deformation vector field. In Figure 10 we show how multiple different constraints can be combined using our representation. First, we can easily require that at a point $p$ the deformation field matches a given vector $v$, by setting $V(p)=v$, in addition to other global constraints. Second, we can find the most isometric (preserving the intrinsic metric) deformation by minimizing $V \mapsto\left\|E^{V}\right\|_{F}^{2}$. At the same time, given a self-map represented as a functional map $S$, we design a symmetric vector field by imposing a constraint of the form $E^{V} C_{S}=C_{S} E^{V}$. Similarly, we can impose an anti-symmetry constraint by requiring $E^{V} C_{S}+C_{S} E^{V}=0$. In comparison, Figure 11 shows an extrinsic deformation design method consisting in projecting each vector field component into the space of symmetric (respectively anti-symmetric) functions. The resulting shapes look quite distorted compared to our solution. Moreover the distance of the conformal shape difference (resp. area-based shape difference) to identity, measuring how far the map $S$ is from an isometry, is of 0.51 (resp. 0.46 ) for our design and 0.63 (resp. 0.56) for the extrinsic design. Thus, the extrinsic deformation design tends to distort the intrinsic structure of the shape. Finally, we test a regularization technique for the deformation field by imposing the commutativity with the

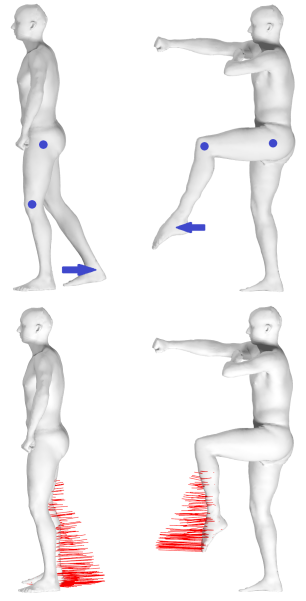

Separate design

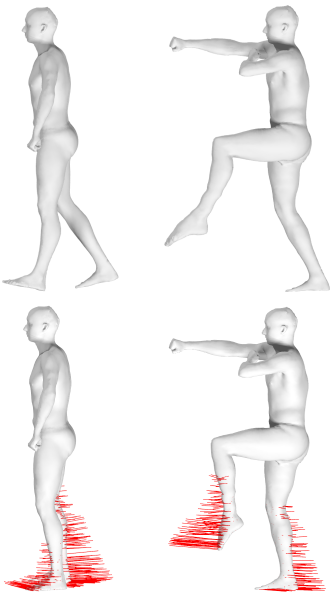

Joint design
Fig. 12: Left: The deformations are designed separately by minimizing the smoothness term $\langle V, \Delta V\rangle$. Right: joint deformation design by adding the commutativity with the mapping to the optimization. Note that the constraints on one shape tend to be transferred to the other.

Laplace-Beltrami operator, which tends to spread to the entire shape. Note that despite the diversity of these constraints, they can all be enforced easily using our operator-based representation. In contrast, the straightforward method shown in Figure 11, consisting in projecting the vector field onto the space of symmetric or anti-symmetric functions, fails in this tasks as it is fully extrinsic.

Figures 12 and 1 present an example of joint deformation design. Namely, we impose a set of directional constraints $U\left(p_{j}\right)=u_{j}$ and $V\left(q_{j}\right)=v_{j}$ on two different shapes $M$ and $N$ and we solve for two deformation fields, one on each shape, that are "informed" by the deformation of the other shape. On a single shape, our objective is designed to promote smoothness of the resulting deformation field and sparsity in the coefficients of the deformation field basis:

$$
\mathbb{E}_{M}(\alpha):=\sum_{i, j} \alpha_{i}\left\langle U_{i}, \Delta_{M} U_{j}\right\rangle \alpha_{j}+\tau\|\alpha\|_{1} .
$$

Therefore, on a single shape, the optimization becomes:

$$
\min _{\alpha} \mathbb{E}_{M}(\alpha), \quad \text { s.t. } \sum_{i} \alpha_{i} U_{i}\left(p_{j}\right)=u_{j},
$$

where the constraints enforce the given pointwise directions. 

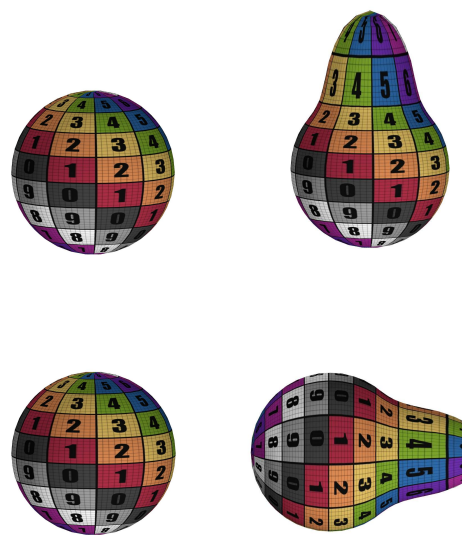

Source

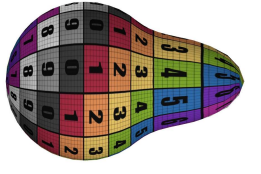

Target
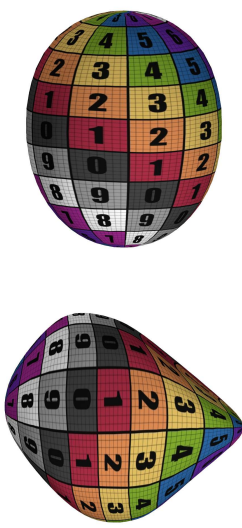

10
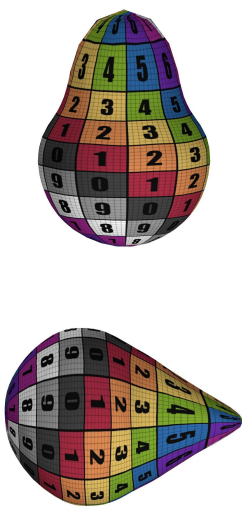

30


60


100

Fig. 13: Two deformation fields (left) resulting from the correspondence encoded via the texture map are represented as operators with increasing number of basis functions and then recovered by solving a least-squares problem. Increasing the size of the basis leads to more accurate representation of high-frequency deformations. Note, our representation can be used to successfully recover almost all deformation fields (up to infinitesimal rigid deformations) including global rotation (bottom row) regardless of the scale of the deformation.

To design the deformation fields jointly, we propose to find a field $U$ on shape $M$ and $V$ on shape $N$ such that for a given functional map $C$ we have $E^{U} C \approx C E^{V}$ while respecting the local constraints on the respective shape. The resulting optimization problem reads:

$$
\begin{aligned}
& \min _{\alpha, \beta}\left\|\sum_{i} \alpha_{i} E^{U_{i}} C-C \sum_{j} \beta_{j} E^{V_{j}}\right\|_{F}^{2}+\mathbb{E}_{M}(\alpha)+\mathbb{E}_{N}(\beta) \\
& \text { s.t. } \sum_{i} \alpha_{i} U_{i}\left(p_{j}\right)=u_{j}, \sum_{i} \beta_{i} V_{i}\left(q_{j}\right)=v_{j} .
\end{aligned}
$$

As a result, the constraints as well as the structure of one shape is transferred onto the other. Moreover the area that could lead to contradictory deformation remains still.

\subsection{Functional Deformation transfer}

Given a deformation field $U$ on shape $M$ represented as an operator and a functional map $C$ from $N$ to shape $M$, we can use our method to transfer the deformation to an arbitrary mesh. The transferred deformation $V=\sum_{i} \alpha_{i} V_{i}$ on shape $N$ by solving:

$$
\min _{\alpha}\left\|E^{U} C-C \sum_{i} \alpha_{i} E^{V_{i}}\right\|^{2}+\tau\|\alpha\|_{1} .
$$

In all of our experiments below, we represent the linear operators $C$ and $E^{V}$ in a reduced functional basis, consisting of the first 200 eigenfunctions of the Laplace-Beltrami operator. We parameterize the space of deformations by computing the 180 extrinsic vector fields in each of the three categories described in Section 8 to build an over-complete dictionnary. This implies that the number of unknowns is relatively small: $\alpha \in \mathbb{R}^{540}$ and is independent of the resolution of the underlying mesh. We choose the parameter $\tau$, controlling the sparsity of the representation, to be $10^{-4}$ times the largest singular value of the linear map $V \mapsto C E^{V}$.

We solve the optimization problem described in Eq. (12) with CVX [Grant and Boyd 2014], using the default approach based on the interior point method.

Using this setup we solve different instances of the deformation transfer problem, namely:



Fig. 14: The deformation field defined by the blue shapes (first column) is transferred to the same shape in different poses (top white shapes). While the style is consistent across the poses (red shapes) some details of the deformation are lost due to the basis representation. The style transfer are compared to the corresponding shape in the collection (bottom white shapes).

- Style transfer: we transfer style across poses. Here, given two different shapes in a rest pose and a deformed version of one of them, we transfer the deformation to the other shape (Figure 14). This also shows that our vector field collection is not limited to a specific type of deformation.

- Symmetry transfer: we transfer a deformation from a shape onto itself using a symmetry map (Figure 15). Note that this task cannot be achieved with standard Jacobian-based methods such as [Sumner and Popović 2004].

We stress that although enabled by our representation, this is by no means the central application and therefore the results presented 

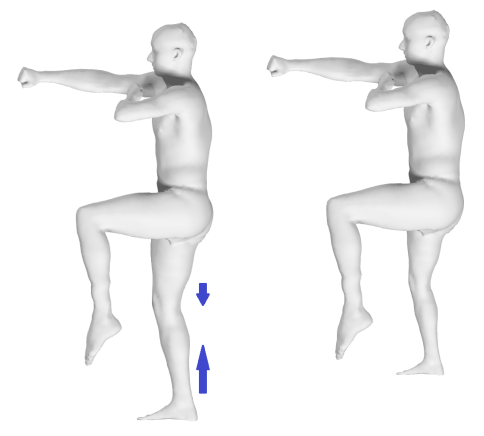

Initial shape

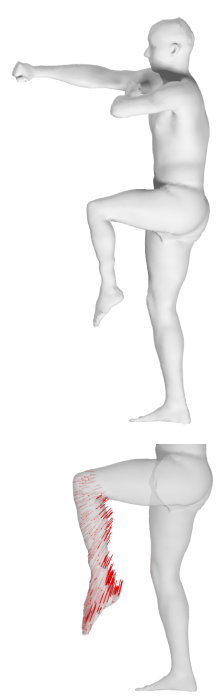

Symmetric Def.
Fig. 15: An initial deformation (first two columns), corresponding to the shrinking of the right leg of a human model, is transferred to the left leg by imposing the commutativity between the infinitesimal shape difference and the symmetry map. Both legs are in different position so the transfer has to adapt to the geometry.

below simply serve as an illustration of the functionality that can be achieved using our functional deformation fields.

Style transfer. We use our approach to transfer style across the poses of different shapes in the Faust dataset [Bogo et al. 2014], shown in Figure 14. Here first consider the deformation field $U$ given by the point displacements across two different shapes in approximately the same reference pose. We then use our framework to transfer $U$ to another shape in a different pose and with different mesh structure. In Figure 14 our method consistently preserves the global structure, although some high frequency details of the deformation are lost due to the projection onto a vector field basis.

Symmetry transfer. One interesting feature of the functional representation of deformation fields is that it is "shape aware." For example in Figure 15 we transfer the shrinking of the right leg to the left leg by looking for the operator which commutes with the operator representation of the symmetry map. Since both legs are in different positions this transfer is not easy to achieve by a simple point-to-point transfer of the vector field or even by transferring it using local coordinates. As shown in Figure 15 bottom row, our transferred deformation field adapts to the geometry.

\subsection{Relation to existing techniques}

An important property of our deformation transfer algorithm is that it relies fully on the deformation of the metric. This makes it fundamentally different from the spectral pose transfer described in [Lévy 2006]. Those methods use the strong stability of the first eigenfunctions of the Laplace-Beltrami under deformation. Thus, a deformation field $V$ can be efficiently transferred by projecting its components into a reduced eigen-basis $\Phi$ of the initial shape and reconstructed using the basis $\Psi$ of the shape to be deformed. The new embedding $X^{\prime}$ is computed from the old embedding $X$ simply by $X^{\prime}:=X+\Psi \Phi^{\top} V$.

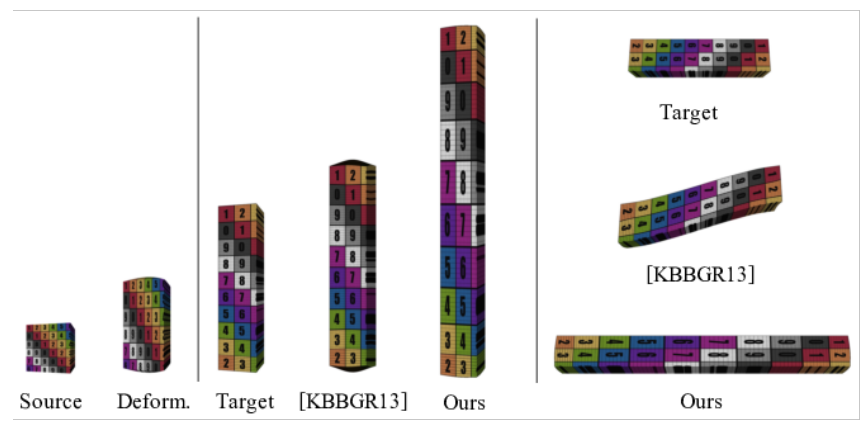

Fig. 16: An initial deformation (first two columns), corresponding to the expansion of a bar is transferred to another longer bar. Using the method described in [Kovnatsky et al. 2013] ([KBBGR13]) the additional height is exactly the same as in the original deformation. Using our method the deformation is indexed on the metric thus the height of the model doubles. Furthermore, our method is rotation invariant (second row).

Recent improvements of this technique [Kovnatsky et al. 2013; Yin et al. 2015] include pre-alignment of the spectral basis but the shortcoming are essentially the same. Figure 16 shows that this deformation transfer is by definition extrinsic, orientation dependent and furthermore completely agnostic to the intrinsic structure of the shape. Our method in contrast is rotation-invariant and directly linked to the induced changes in the geometry.

We also compare our method with the algorithm for deformation transfer described in [Sumner and Popović 2004]. This method is based on reallocating Jacobian matrices defined on triangles of the source mesh to those of the target mesh. This method, however, is not without limitations. First, this transfer does not take into account changes in orientation from the source to the target thus ruling out any possibility of symmetric transfer and requiring a pre-alignment of the source and target meshes. This can be challenging to achieve in practice in case of non-rigid deformations (e.g. Figure 15). Secondly, it assumes as input a triangle-to-triangle map which can be cumbersome to obtain.

These limitations do not apply to our representation as our approach is based on transferring metric information, and is therefore immune to changes of orientation. Moreover, instead of a triangleto-triangle map, an approximate functional map is enough. Furthermore, note that although in general reconstructing geometry from metric tensors is more difficult than a reconstruction from Jacobians as local rotations are no longer available (see e.g. [Boscaini et al. 2015]) our method relies on solving a moderately-sized convex optimization problem.

Figure 17 shows that working within the functional map framework makes our algorithm more robust to noise usually encounter when using this representation. The computation of functional maps, as described in [Ovsjanikov et al. 2012], is done by solving a least squares system incorporating intrinsic descriptors (HKS, WKS) therefore there often exists multiple solutions in presence of an intrinsic symmetry $\pi$. We model a noisy functional map $C^{\tau}$ by a linear blending between the direct map (mapping the left to the left and the right to the right) and the anti-symmetric map (mapping the left to the right and the right to the left) represented as operators:

$$
C^{\tau}=\tau C_{\varphi \circ \pi}+(1-\tau) C_{\varphi}
$$

Our method outputs a non-linear interpolation between the deformation and its symmetric version while the method by Sumner et al. exhibits various artifacts. 


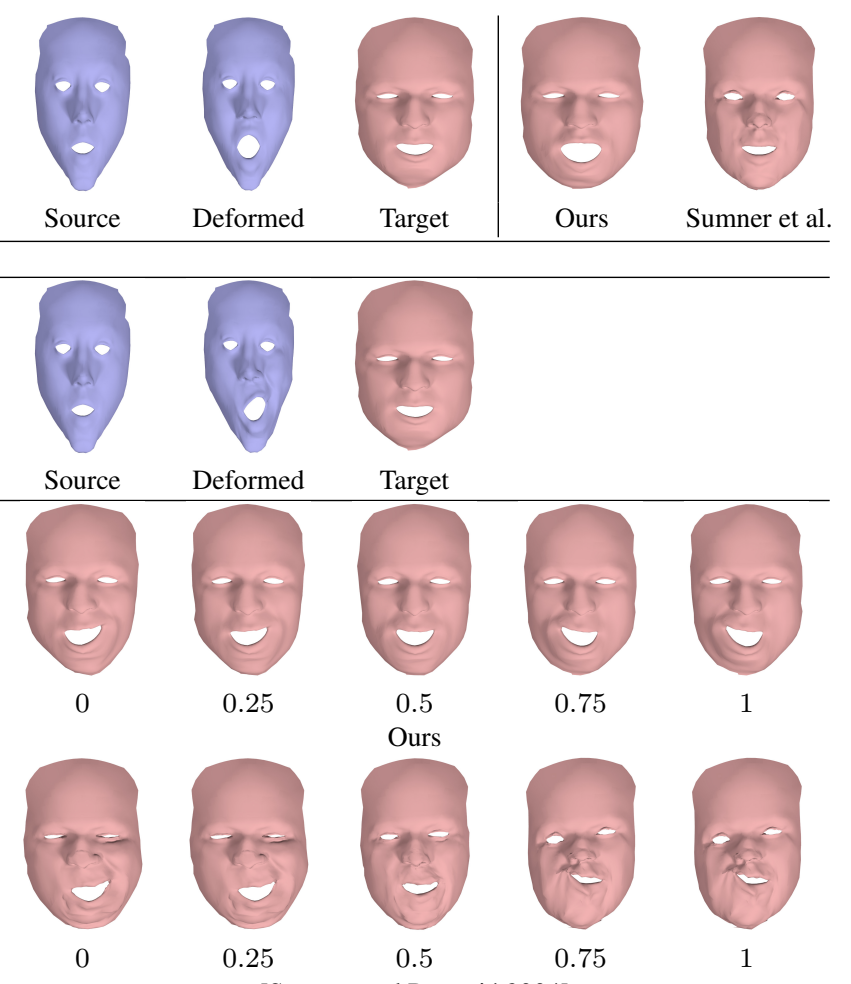

[Sumner and Popović 2004]

Fig. 17: Deformation transfer using a noisy functional map consisting of a linear blending between the direct functional map $(\tau=0)$ and the symmetric functional map ( $\tau=1$ ). Our method is robust to this noise and outputs a non-linear interpolation of the deformation and its symmetric version. The method of in [Sumner and Popović 2004] fails when provided with a triangleto-triangle map resulting of the conversion of the noisy functional map.

\section{CONCLUSION AND FUTURE WORK}

In this paper we presented a method for representing extrinsic vector fields as linear operators acting on functions on the shapes, by considering the metric distortion induced by the deformation. In particular, we base our representation on the infinitesimal strain tensor and show how it leads to a linear functional operator that can naturally be combined with other such operators including functional maps and the Laplace-Beltrami. We showed how this representation can be used to analyze and design deformations and to introduce extrinsic information into the computation of functional correspondences. In the future, we are planning to use the newly introduced functional representation for shape animation. In this context, it would be interesting to establish a connection between the metric on the space of functional deformation fields and different inner products as suggested in [Eckstein et al. 2007]. It would also very interesting to use our representation within the framework of shape spaces, e.g. [Kilian et al. 2007], for exploration and design.

\section{REFERENCES}

Adams, B., Ovsjanikov, M., Wand, M., Seidel, H.-P., And Guibas, L. J. 2008. Meshless modeling of deformable shapes and their motion. In Proceedings of the 2008 ACM SIGGRAPH/Eurographics Symposium on Computer Animation. Eurographics Association, 77-86.

Azencot, O., Ben-Chen, M., Chazal, F., And Ovsjanikov, M. 2013. An operator approach to tangent vector field processing. In Computer
Graphics Forum. Vol. 32. 73-82.

Azencot, O., Corman, E., Ben-Chen, M., And Ovsjanikov, M. 2017. Consistent functional cross field design for mesh quadrangulation. $A C M$ Trans. Graph. 36, 4, 92:1-92:13.

Azencot, O., Ovsjanikov, M., Chazal, F., And Ben-Chen, M. 2015. Discrete derivatives of vector fields on surfaces-an operator approach ACM Transactions on Graphics (TOG) 34, 3, 29.

Baran, I., Vlasic, D., Grinspun, E., And Popović, J. 2009. Semantic deformation transfer. In ACM Transactions on Graphics (TOG). Vol. 28 ACM, 36.

Bogo, F., ROMERO, J., LOPER, M., AND BLACK, M. J. 2014. FAUST: Dataset and evaluation for 3D mesh registration. In Proceedings IEEE Conf. on Computer Vision and Pattern Recognition (CVPR). IEEE, Piscataway, NJ, USA.

Boscaini, D., Eynard, D., Kourounis, D., And Bronstein, M. M. 2015. Shape-from-operator: Recovering shapes from intrinsic operators. In Computer Graphics Forum. Vol. 34. Wiley Online Library, 265-274.

Botsch, M., Kobbelt, L., Pauly, M., Alliez, P., AND Lévy, B. 2010. Polygon mesh processing. CRC press.

BOTSCH, M. AND SORKINE, O. 2008. On linear variational surface deformation methods. Visualization and Computer Graphics, IEEE Transactions on 14, 1, 213-230.

Celniker, G. AND Gossard, D. 1991. Deformable curve and surface finite-elements for free-form shape design. In ACM SIGGRAPH computer graphics. Vol. 25. ACM, 257-266.

CIARLET, P. G. 2000. Theory of shells. Vol. 3. Elsevier.

Crane, K., Pinkall, U., AND SChröder, P. 2011. Spin transformations of discrete surfaces. In ACM Transactions on Graphics (TOG). Vol. 30. ACM, 104.

DEY, T. K., RANJAN, P., AND WANG, Y. 2012. Eigen deformation of 3d models. The Visual Computer 28, 6-8, 585-595.

Do CARMo, M. 2013. Riemannian Geometry. Mathematics: Theory \& Applications. Birkhäuser Boston.

Eckstein, I., Pons, J.-P., Tong, Y., Kuo, C.-C., And Desbrun, M. 2007. Generalized surface flows for mesh processing. In Proceedings of the fifth Eurographics symposium on Geometry processing. Eurographics Association, 183-192.

Eigensatz, M. And Pauly, M. 2009. Positional, metric, and curvature control for constraint-based surface deformation. In Computer Graphics Forum. Vol. 28. Wiley Online Library, 551-558.

GRANT, M. AND BOYD, S. 2014. CVX: Matlab software for disciplined convex programming, version 2.1. http://cvxr. com/cvx.

GusKov, I., Sweldens, W., AND SCHRÖDER, P. 1999. Multiresolution signal processing for meshes. In Proceedings of the 26th Annual Conference on Computer Graphics and Interactive Techniques. SIGGRAPH '99. 325-334.

Kilian, M., Mitra, N. J., And Pottmann, H. 2007. Geometric modeling in shape space. ACM Transactions on Graphics (TOG) 26, 3, 64.

Kobbelt, L., Campagna, S., Vorsatz, J., And Seidel, H.-P. 1998. Interactive multi-resolution modeling on arbitrary meshes. In Proceedings of the 25th Annual Conference on Computer Graphics and Interactive Techniques. SIGGRAPH '98. 105-114.

Kovnatsky, A., Bronstein, M. M., Bronstein, A. M., Glashoff, K., AND KIMMEL, R. 2013. Coupled quasi-harmonic bases. In Computer Graphics Forum. Vol. 32. Wiley Online Library, 439-448.

LÉVY, B. 2006. Laplace-beltrami eigenfunctions towards an algorithm that "understands" geometry. In Proceedings of the IEEE International Conference on Shape Modeling and Applications.

Lipman, Y., Sorkine, O., Cohen-Or, D., Levin, D., Rossi, C., AND SEIDEL, H.-P. 2004. Differential coordinates for interactive mesh editing. In Shape Modeling Applications. 181-190. 
Litany, O., Rodolà, E., Bronstein, A. M., And Bronstein, M. M. 2017. Fully spectral partial shape matching. In Computer Graphics Forum. Vol. 36. Wiley Online Library, 247-258.

LiU, B., Tong, Y., Goes, F. D., And Desbrun, M. 2016. Discrete connection and covariant derivative for vector field analysis and design. ACM Transactions on Graphics (TOG) 35, 3, 23.

Martinez Esturo, J., Rössl, C., AND Theisel, H. 2013. Generalized metric energies for continuous shape deformation. Springer LNCS (Proc. Curves and Surfaces 2012) 8177, 1, 135-157.

Müller, M., Chentanez, N., KIM, T.-Y., And Macklin, M. 2014 Strain based dynamics. In Proc. SCA. 149-157.

Nealen, A., Müller, M., Keiser, R., Boxerman, E., and Carlson, M. 2006. Physically based deformable models in computer graphics. In Computer graphics forum. Vol. 25. Wiley Online Library, 809-836.

Nealen, A., Sorkine, O., Alexa, M., And Cohen-Or, D. 2005. A sketch-based interface for detail-preserving mesh editing. ACM Transactions on Graphics (Proceedings of ACM SIGGRAPH) 24, 3, 1142-1147.

Ovsjanikov, M., Ben-Chen, M., SOlomon, J., Butscher, A., AND GuIBAS, L. 2012. Functional maps: a flexible representation of maps between shapes. ACM Transactions on Graphics (TOG) 31, 4, 30.

Ovguanikov, M., Corman, E., Bronstein, M., Rodolà, E., BenChen, M., Guibas, L., Chazal, F., And Bronstein, A. 2016. Computing and processing correspondences with functional maps. In $S I G$ GRAPH ASIA 2016 Courses. ACM, 9.

Panozzo, D., Puppo, E., Tarini, M., And Sorkine-Hornung, O. 2014. Frame fields: Anisotropic and non-orthogonal cross fields. $A C M$ Transactions on Graphics (proceedings of ACM SIGGRAPH) 33, 4, 134:1134:11.

Paries, N., Degener, P., And Klein, R. 2007. Simple and efficient mesh editing with consistent local frames. In Computer Graphics and Applications, 2007. PG'07. 15th Pacific Conference on. IEEE, 461-464.

Pavlov, D., Mullen, P., Tong, Y., Kanso, E., Marsden, J. E., And DESBRUN, M. 2011. Structure-preserving discretization of incompressible fluids. Physica D: Nonlinear Phenomena 240, 6, 443-458.

Pokrass, J., Bronstein, A. M., Bronstein, M. M., Sprechmann, P., AND SAPIRO, G. 2013. Sparse modeling of intrinsic correspondences. In Computer Graphics Forum. Vol. 32. Wiley Online Library, 459-468.

RUMPF, M. AND WARDETZKY, M. 2014. Geometry processing from an elastic perspective. GAMM-Mitteilungen 37, 2, 184-216.

Rustamov, R. M., Ovsjanikov, M., Azencot, O., Ben-Chen, M., ChAZAL, F., AND GuiBAs, L. 2013. Map-based exploration of intrinsic shape differences and variability. ACM Trans. Graph. 32, 4, 72:1-72:12.

Sela, M., Aflalo, Y., AND Kimmel, R. 2015. Computational caricaturization of surfaces. Computer Vision and Image Understanding 141, $1-17$.

Solomon, J., Ben-Chen, M., Butscher, A., And Guibas, L. 2011. As-killing-as-possible vector fields for planar deformation. In Computer Graphics Forum. Vol. 30. Wiley Online Library, 1543-1552.

Sorkine, O. AND ALEXA, M. 2007. As-rigid-as-possible surface modeling. In Proceedings of EUROGRAPHICS/ACM SIGGRAPH Symposium on Geometry Processing. 109-116.

Sorkine, O., Cohen-Or, D., Lipman, Y., AleXa, M., Rössl, C., AND SEIDEL, H.-P. 2004. Laplacian surface editing. In Proc. SGP. ACM, 175-184.

Sumner, R. W. AND Popović, J. 2004. Deformation transfer for triangle meshes. In ACM Transactions on Graphics (TOG). Vol. 23. 399-405.

Terzopoulos, D., Platt, J., Barr, A., AND Fleischer, K. 1987. Elastically deformable models. In ACM Siggraph Computer Graphics. Vol. 21. ACM, 205-214.
Thomaszewski, B., Pabst, S., and Strasser, W. 2009. Continuumbased strain limiting. In Computer Graphics Forum. Vol. 28. Wiley Online Library, 569-576.

Tombari, F., Salti, S., AND Di Stefano, L. 2010. Unique signatures of histograms for local surface description. In European conference on computer vision. Springer, 356-369.

VAllet, B. AND LÉvy, B. 2008. Spectral geometry processing with manifold harmonics. Comput. Graph. Forum 27, 2, 251-260.

Vaxman, A., Müller, C., AND Weber, O. 2015. Conformal mesh deformations with möbius transformations. ACM Transactions on Graphics (TOG) 34, 4, 55 .

VON Funck, W., Theisel, H., AND SEIDEL, H.-P. 2006. Vector field based shape deformations. In ACM Transactions on Graphics (TOG) Vol. 25. ACM, 1118-1125.

Von-Tycowicz, C., Schulz, C., Seidel, H.-P., ANd Hildebrandt, K. 2015. Real-time nonlinear shape interpolation. ACM Transactions on Graphics (TOG) 34, 3, 34.

WELCH, W. AND WITKIN, A. 1992. Variational surface modeling. In ACM SIGGRAPH computer graphics. Vol. 26. ACM, 157-166.

YIN, M., LI, G., LU, H., OUYANG, Y., ZHANG, Z., AND XIAN, C. 2015. Spectral pose transfer. Computer Aided Geometric Design 35, 82-94.

Yu, Y., Zhou, K., Xu, D., SHI, X., BaO, H., Guo, B., AND SHum, H.-Y. 2004. Mesh editing with poisson-based gradient field manipulation. $A C M$ Transactions on Graphics (TOG) 23, 3, 644-651.

ZaYer, R., RÖssl, C., KARNI, Z., AND SEIDEl, H.-P. 2005. Harmonic guidance for surface deformation. In Computer Graphics Forum. Vol. 24 Wiley Online Library, 601-609.

Zhang, Z., LI, G., LU, H., OUYAng, Y., Yin, M., AND XIAN, C. 2015. Fast as-isometric-as-possible shape interpolation. Computers \& Graphics 46, 244-256.

Zheng, Q., HaO, Z., HuAng, H., Xu, K., Zhang, H., CoHen-OR, D., AND CHEN, B. 2015. Skeleton-intrinsic symmetrization of shapes. In Computer Graphics Forum. Vol. 34. Wiley Online Library, 275-286.

ZORIN, D., SCHRÖDER, P., AND SWELDENS, W. 1997. Interactive multiresolution mesh editing. In Proc. SIGGRAPH. 259-268. 


\section{Online Appendix to:}

\section{Functional Characterization of Infinitesimal Deformation}

\section{ETIENNE CORMAN and MAKS OVSJANIKOV \\ LIX, École Polytechnique, CNRS}

\section{EXTRINSIC VECTOR FIELDS AS OPERATOR}

PRoposition 1. For any extrinsic vector field $V$ there is a unique linear functional operator $E^{V}$ that satisfies:

$$
\int_{M}\left\langle\nabla g, \nabla E^{V}(f)\right\rangle \mathrm{d} \mu=\int_{M} \mathcal{L}_{V} \mathbf{g}(\nabla g, \nabla f) \mathrm{d} \mu .
$$

Moreover, this operator is linear in both the vector field $V$ and function $f$.

Proof. Let $H_{0}^{1}(M)$ be the space of square integrable functions with $L^{2}(M)$ gradients and zero integrals:

$H_{0}^{1}(M)=\left\{f \in L^{2}(M): \int_{M}\|\nabla f\|^{2} \mathrm{~d} \mu<+\infty, \int_{M} f \mathrm{~d} \mu=0\right\}$.

This space seems natural when studying this operator as $E^{V}$ maps any constant function to zero. When equipped of the scalar product $\langle., .\rangle_{L^{2}}+\langle., .\rangle_{H_{0}^{1}}, H_{0}^{1}$ is a Hilbert space.

The bilinear form $(f, g) \mapsto\langle f, g\rangle_{H_{0}^{1}(M)}$ is continuous and coercive thanks to the Wirtinger's inequality [Brezis 2010]. Moreover, for a given function $g$ in $H_{0}^{1}(M)$ the linear form $f \mapsto$ $\int_{M} \mathcal{L}_{V} \mathbf{g}(\nabla g, \nabla f) \mathrm{d} \mu$ is continuous assuming that $V$ is smooth enough (at least $H_{1}$ ) since we have the inequality:

$$
\int_{M} \mathcal{L}_{V} \mathbf{g}(\nabla g, \nabla f) \mathrm{d} \mu \leq\|\nabla g\|_{L^{2}}^{2}\left\|\mathcal{L}_{V} \mathbf{g}\right\|_{L^{2}}^{2}\|\nabla f\|_{L^{2}}^{2}
$$

Thus all conditions of the Lax-Milgram theorem [Brezis 2010] are satisfied therefore for any function $g$ there exists a unique $E^{V}(g)$ satisfying Eq. (1).

\section{RELATION TO SHAPE DIFFERENCE OPERATORS}

\subsection{Unified Shape Difference}

Definition 2. Assuming that $\varphi: N \rightarrow M$ is a diffeomor phism, the unified shape difference $D_{I}: \mathcal{C}^{\infty}(M) \rightarrow \mathcal{C}^{\infty}(M)$ is

Permission to make digital or hard copies of part or all of this work for personal or classroom use is granted without fee provided that copies are not made or distributed for profit or commercial advantage and that copies show this notice on the first page or initial screen of a display along with the full citation. Copyrights for components of this work owned by others than ACM must be honored. Abstracting with credit is permitted. To copy otherwise, to republish, to post on servers, to redistribute to lists, or to use any component of this work in other works requires prior specific permission and/or a fee. Permissions may be requested from Publications Dept., ACM, Inc., 2 Penn Plaza, Suite 701, New York, NY 10121-0701 USA, fax +1 (212) 869-0481, or permissions@acm.org.

(c) YYYY ACM 0730-0301/YYYY/17-ARTXXX \$10.00

DOI 10.1145/XXXXXXX.YYYYYYY

http://doi.acm.org/10.1145/XXXXXXX.YYYYYYY defined implicitly by:

$$
\left\langle f, D_{I}(g)\right\rangle_{H_{0}^{1}(M)}:=\int_{M} C_{\varphi^{-1}}\left(\left\langle\nabla C_{\varphi}(f), \nabla C_{\varphi}(g)\right\rangle\right) \mathrm{d} \mu^{M} .
$$

Suppose that $\varphi: N \rightarrow M$ is a diffeomorphism. We denote $\left(\varphi_{\star} X\right)_{\varphi(p)}=\mathrm{d} \varphi_{p} X_{p}$ the pullback of a vector field and $\mathrm{d} \varphi_{p}: T_{p} N \rightarrow T_{\varphi(p)} M$ the linear map between tangent spaces. Moreover the pullback with respect to $\varphi^{-1}$ of the metric field $\mathbf{g}^{N}: T_{p} N \times T_{p} N \rightarrow \mathbb{R}$ is given by $\left(\left(\varphi^{-1}\right)^{\star} \mathbf{g}^{N}\right)_{p}(X, Y)=$ $\mathrm{g}_{\varphi^{-1}(p)}^{N}\left(\mathrm{~d} \varphi^{-1} X, \mathrm{~d} \varphi^{-1} Y\right)$. For the gradient of a function $f$ on $\bar{M}$ at a point $q \in N$ :

$$
\nabla(f \circ \varphi)_{q}=\left(\mathrm{d} \varphi^{-1} \nabla f\right) \circ \varphi(q)
$$

Let's note $\varphi^{-1}(p)=q \in N$, therefore the pullback metric reads

$$
\begin{aligned}
\langle\nabla(f \circ \varphi), \nabla(g \circ \varphi)\rangle_{q} & =\mathbf{g}_{\varphi(q)}^{N}\left(\mathrm{~d} \varphi^{-1} \nabla f, \mathrm{~d} \varphi^{-1} \nabla g\right) \\
& =\left(\left(\varphi^{-1}\right)^{\star} \mathbf{g}^{N}\right)_{\varphi(q)}(\nabla f, \nabla g) .
\end{aligned}
$$

We can now rewrite Definition 2 with respect to the pullback metric:

$$
\begin{aligned}
\int_{M}\left\langle\nabla f, \nabla D_{I}(g)\right\rangle \mathrm{d} \mu & =\int_{M} C_{\varphi}^{-1}\left(\left\langle\nabla C_{\varphi}(f), \nabla C_{\varphi}(g)\right\rangle\right) \mathrm{d} \mu \\
& =\int_{M} C_{\varphi}^{-1}\left(\left(\left(\varphi^{-1}\right)^{\star} \mathbf{g}^{N}\right)_{\varphi(p)}(\nabla f, \nabla g)\right) \mathrm{d} \mu \\
& =\int_{M}\left(\left(\varphi^{-1}\right)^{\star} \mathbf{g}^{N}\right)(\nabla f, \nabla g) \mathrm{d} \mu
\end{aligned}
$$

This alternative definition leads to the characterization of the metric change:

Proposition 2. $D_{I}(f)=f$ for all $f \in \mathcal{C}^{\infty}(M)$ if and only if $\varphi$ is an isometry.

Proof. If $\varphi$ is an isometry then $\left(\varphi^{-1}\right)^{\star} \mathbf{g}^{N}=\mathbf{g}^{M}$ so

$$
\int_{M}\left\langle\nabla f, \nabla D_{I}(g)\right\rangle \mathrm{d} \mu^{M}=\int_{M}\langle\nabla f, \nabla g\rangle \mathrm{d} \mu
$$

Using the fundamental lemma of calculus of variations: $\Delta D_{I}(g)=$ $\Delta g$.

If $D_{I}(f)=f$ then

$$
\int_{M}\langle\nabla f, \nabla g\rangle \mathrm{d} \mu=\int_{M}\left(\left(\varphi^{-1}\right)^{\star} \mathbf{g}^{N}\right)(\nabla f, \nabla g) \mathrm{d} \mu
$$

Using a result from [Schumacher 2013], it implies $\mathbf{g}^{M}=$ $\left(\varphi^{-1}\right)^{\star} \mathbf{g}^{N}$ so $\varphi$ is an isometry. $\square$

\subsection{Infinitesimal Shape Difference Operators}

To define the infinitesimal shape differences we first need to introduce the correct framework and notation. Let's assume that the

ACM Transactions on Graphics, Vol. VV, No. N, Article XXX, Publication date: Month YYYY. 
family of oriented surfaces $M_{t}$ without boundary of intrinsic dimension 2 are isometrically immersed in $\mathbb{R}^{3}$ by the local mappings $F_{t}: U \subset \mathbb{R}^{2} \rightarrow M_{t} \subset \mathbb{R}^{3}$. This family of manifolds is generated by the displacement of the points along the smooth vector field $V(p) \in T_{p} M \times T_{p} M^{\perp} \simeq \mathbb{R}^{3}$ :

$$
\frac{\partial F_{t}}{\partial t}(p)=V(p), \quad(p, t) \in M \times \mathbb{R}^{+}
$$

The metric of the embedded surface is by definition $\mathbf{g}_{i j}^{t}=$ $\left\langle\partial_{i} F_{t}, \partial_{j} F_{t}\right\rangle$ and the area form is $\mu^{t}=\sqrt{\operatorname{det} \mathbf{g}^{t}}$. The Riemannian connection on the ambient space $\mathbb{R}^{3}$ is denoted $\bar{\nabla}$. As mentioned in [do Carmo 2013], the projection of the ambient connection into the tangent space of $M$ coincides the unique Levi-Civita connection on $M$. Therefore the connection $\nabla$ on $M$ is naturally extended to extrinsic vector fields by $\nabla_{i} V_{j}=\left\langle\partial_{i} V, \partial_{j} F_{t}\right\rangle$. Once the connection is defined other differential operator can extended to extrinsic vector fields for example the divergence is defined as the trace of the connection $\operatorname{div}(V)=\mathbf{g}^{i j} \nabla_{i} V_{j}$

We consider the family of diffeomorphisms $\varphi_{t}: M_{t} \rightarrow M$ given by $\varphi_{t}(p): F_{t}-t(p) V(p)$.

The derivative of local quantities links the Lie derivative with the Strain tensor.

LEMMA 1. Given a one parameter family of surfaces described in Eq. (2), for a fixed point p, the first-order change in the metric tensor $\mathbf{g}$ and in the local area element $\mu=\sqrt{\operatorname{det}(\mathbf{g})}$ are given as:

$$
\begin{aligned}
& \left.\frac{\partial \mathbf{g}(t)}{\partial t}\right|_{t=0}=\mathcal{L}_{V} \mathbf{g} \\
& \left.\frac{\partial \mu(t)}{\partial t}\right|_{t=0}=\operatorname{div}(V) \mu .
\end{aligned}
$$

Proof. Those properties are easily proven when using local coordinates. Given a family of diffeomorphisms $\varphi_{t}$ the Lie derivative of the metric tensor with respect to the vector field $V$ denoted $\mathcal{L}_{V} \mathbf{g}$ is by definition:

$$
\mathcal{L}_{V} \mathbf{g}:=\left.\frac{\partial}{\partial t}\left(\left(\varphi_{t}^{-1}\right)^{\star} \mathbf{g}(t)\right)\right|_{t=0} .
$$

Since the local immersion $F_{t}$ use a common chart system, the coordinates of the pullback metric $\left(\left(\varphi_{t}^{-1}\right)^{\star} \mathbf{g}^{t}\right)_{i j}$ are equal to the metric on $M_{t}$ in local coordinates $\mathbf{g}_{i j}(t)=\left\langle\partial_{i} F_{t}, \partial_{j} F_{t}\right\rangle$. The computation of derivative is then straigthforward:

$$
\begin{aligned}
\left.\frac{\partial}{\partial t}\left(\left(\left(\varphi_{t}^{-1}\right)^{\star} \mathbf{g}(t)\right)_{i j}\right)\right|_{t=0} & =\left.\frac{\partial}{\partial t}\left(\left\langle\partial_{i} F_{t}, \partial_{j} F_{t}\right\rangle\right)\right|_{t=0} \\
& =\nabla_{i} V_{j}+\nabla_{j} V_{i}
\end{aligned}
$$

From there, Eq. (4) is easily obtained:

$$
\begin{aligned}
\left.\frac{\partial \mu(t)}{\partial t}\right|_{t=0} & =\left.\frac{\partial}{\partial t}(\sqrt{\operatorname{det}(\mathbf{g}(t))})\right|_{t=0} \\
& =\frac{1}{2 \mu} \operatorname{det}(\mathbf{g}) \mathbf{g}^{i j}\left(\nabla_{i} V_{j}+\nabla_{j} V_{i}\right)=\operatorname{div}(V) \mu
\end{aligned}
$$

We then obtain the derivative of the shape differences.

Proposition 3. Let $V$ be a smooth deformation field on $M$, the derivatives of $D_{A}, D_{C}$ and $D_{I}$ at time zero satisfy for all smooth

ACM Transactions on Graphics, Vol. VV, No. N, Article XXX, Publication date: Month YYYY. functions $f, g$ :

$$
\begin{aligned}
\left\langle f, E_{A}^{V}(g)\right\rangle_{L^{2}}^{M} & =\int_{M} \operatorname{div}(V) f g \mathrm{~d} \mu, \\
\left\langle f, E_{C}^{V}(g)\right\rangle_{H_{0}^{1}}^{M} & =\int_{M} \operatorname{div}(V)\langle\nabla f, \nabla g\rangle-\mathcal{L}_{V} \mathbf{g}(\nabla f, \nabla g) \mathrm{d} \mu, \\
\left\langle f, E_{I}^{V}(g)\right\rangle_{H_{0}^{1}}^{M} & =-\int_{M} \mathcal{L}_{V} \mathbf{g}(\nabla f, \nabla g) \mathrm{d} \mu .
\end{aligned}
$$

PROOF. The first statement is obtained by using (4):

$$
\begin{aligned}
\left\langle f, \partial_{t} D_{A}(g)\right\rangle_{L^{2}} & =\left.\frac{\partial}{\partial t}\left(\int_{M_{t}} C_{t}(f) C_{t}(g) \mathrm{d} \mu^{t}\right)\right|_{t=0} \\
& =\left.\frac{\partial}{\partial t}\left(\int_{M} f g \mathrm{~d}\left(\left(\varphi_{t}\right)_{\star} \mu^{t}\right)\right)\right|_{t=0} \\
& =\int_{M} \operatorname{div}(V) f g \mathrm{~d} \mu
\end{aligned}
$$

For the second statement let's start with the evolution of the point-wise scalar product between gradient:

$$
\begin{aligned}
\left.\frac{\partial}{\partial t}(\langle\nabla f, \nabla g\rangle)\right|_{t=0} & =\left.\frac{\partial}{\partial t}\left(\mathbf{g}^{i k} \partial_{k} f\right) \mathbf{g}_{i j}\left(\mathbf{g}^{j l} \partial_{l} g\right)\right|_{t=0} \\
& =\left.\frac{\partial}{\partial t} \partial_{i} f \mathbf{g}^{i j} \partial_{j} g\right|_{t=0} \\
& =-\left.\left(\mathbf{g}^{i k} \partial_{k} f\right) \frac{\partial \mathbf{g}_{i j}}{\partial t}\right|_{t=0}\left(\mathbf{g}^{j l} \partial_{l} g\right) \\
& =-\left\langle\nabla f, \nabla_{\nabla g} V\right\rangle-\left\langle\nabla_{\nabla f} V, \nabla g\right\rangle
\end{aligned}
$$

It follows that:

$$
\begin{aligned}
\left\langle f, \partial_{t} D_{C}(g)\right\rangle_{H_{0}^{1}} & =\left.\frac{\partial}{\partial t}\left(\int_{M_{t}}\left\langle\nabla C_{t}(f), \nabla C_{t}(g)\right\rangle \mathrm{d} \mu^{t}\right)\right|_{t=0} \\
& =\left.\frac{\partial}{\partial t}\left(\int_{M}\left(\left(\varphi_{t}^{-1}\right)^{\star} \mathbf{g}^{t}\right)(\nabla f, \nabla g) \mathrm{d}\left(\left(\varphi_{t}\right)_{\star} \mu^{t}\right)\right)\right|_{0} \\
& =\int_{M} \operatorname{div}(V)\langle\nabla f, \nabla g\rangle \mathrm{d} \mu \\
& -\int_{M}\left(\left\langle\nabla f, \nabla_{\nabla g} V\right\rangle+\left\langle\nabla_{\nabla f} V, \nabla g\right\rangle\right) \mathrm{d} \mu
\end{aligned}
$$

Starting from Definition 2:

$$
\begin{aligned}
\left\langle f, \partial_{t} D_{I}(g)\right\rangle_{H_{0}^{1}} & =\left.\frac{\partial}{\partial t}\left(\int_{M} C_{t}^{-1}\left(\left\langle\nabla C_{t}(f), \nabla C_{t}(g)\right\rangle\right) \mathrm{d} \mu\right)\right|_{t=0} \\
& =\left.\frac{\partial}{\partial t}\left(\int_{M}\left(\left(\varphi_{t}^{-1}\right)^{\star} \mathbf{g}^{t}\right)(\nabla f, \nabla g) \mathrm{d} \mu\right)\right|_{t=0} \\
& =-\int_{M}\left\langle\nabla f, \nabla_{\nabla g} V\right\rangle+\left\langle\nabla_{\nabla f} V, \nabla g\right\rangle \mathrm{d} \mu
\end{aligned}
$$

\section{DISCRETE CONNECTION}

The connection of the ambient space $\bar{\nabla}_{u} V$ where $u$ is a tangent vector and $V$ is an extrinsic vector field :

$$
\begin{aligned}
\bar{\nabla}: \mathbb{R}^{3|\mathcal{F}|} \times \mathbb{R}^{3|\mathcal{V}|} & \rightarrow \mathbb{R}^{3|\mathcal{F}|} \\
(u, V) & \mapsto \bar{\nabla}_{u} V
\end{aligned}
$$


Recall that we build the connection $\bar{\nabla}$ using finite differences as follows. Since extrinsic vector fields are defined at vertices the differences are taken along the edges.

DEFINITION 3. In a given triangle $T \in \mathcal{F}$ the ambient covariant derivative along the edge $e_{i j}$ is defined by

$$
\left(\bar{\nabla} \underset{e_{i j}}{\left\|e_{i j}\right\|} V\right)_{T}=\frac{V_{i}-V_{j}}{\left\|e_{i j}\right\|} .
$$

Thus the ambient connection in the directions $E=\left(e_{i j}, e_{j k}\right)$ can be stored in a matrix

$$
\left(\bar{\nabla}_{E} V\right)_{T}=\left(V_{i}-V_{j} V_{j}-V_{k}\right) .
$$

Then, given any tangent vector $x=E \alpha$, the covariant derivative in its direction can be computed as $\bar{\nabla}_{x} V=\left(\bar{\nabla}_{E} V\right) \alpha$.

Given the expression above, the discrete Lie derivative of the metric at triangle $T$ follows immediately. Namely for any pair of tangent vectors $x=E \alpha, y=E \beta$ in the triangle $T$, we have:

$$
\mathcal{L}_{V} \mathbf{g}(x, y)_{T}=\left\langle x,\left(\bar{\nabla}_{E} V\right) \beta\right\rangle+\left\langle\left(\bar{\nabla}_{E} V\right) \alpha, y\right\rangle .
$$

After integration we obtain the discrete infinitesimal shape difference:

$$
f^{\top} W_{M} E^{V} g=-\sum_{T \in \mathcal{F}} \mathcal{L}_{V} \mathbf{g}(\nabla f, \nabla g)_{T} \mu(T) .
$$

The expression of the matrix $W_{M} E^{V}$ is more easily found using the derivative of the unified shape difference operator (see Section 4 ) and is proven later in Thm. 1. The same goes for the proof of Prop. 4 which is also postponed until Section 4.4.

\section{DISCRETE INFINITESIMAL SHAPE DIFFERENCES}

\subsection{Discrete Unified Shape Differences}

The discretization of the unified shape difference is straightforward when $N$ and $M$ are triangle meshes and share the same connectivity. In Definition 2 given above, the gradients and the point-wise scalar products are taken on $N$ while the measure $\mathrm{d} \mu^{M}$ comes from $M$. Therefore the right hand side can be discretized by a modified cotangent weight formula:

$$
\begin{aligned}
W_{M} D_{I} & =W_{N}^{M}, \text { where } \\
\left(W_{N}^{M}\right)_{i, j} & =\frac{1}{2}\left(\frac{\mu^{M}\left(T_{\alpha}\right)}{\mu^{N}\left(T_{\alpha}\right)} \cot \alpha_{i j}^{N}+\frac{\mu^{M}\left(T_{\beta}\right)}{\mu^{N}\left(T_{\beta}\right)} \cot \beta_{i j}^{N}\right) .
\end{aligned}
$$

In Section 4.1 we derived an infinitesimal shape difference from a discrete connection. This discrete can be done by a time derivative of the Eq. (6). To do so, however, we need to introduce an alternative formalism for the cotangent-weights Laplacian.

\subsection{Cotangent weights alternative}

The usual cotangent weight formula is not well-suited to carry out the computations. Therefore we use an alternative formulation which makes more apparent the link with continuous properties.

We denote the local basis $E=\left(e_{i j}, e_{j k}\right)$ formed by edges of triangle of a triangle $T=\left\{x_{i}, x_{j}, x_{k}\right\} \in \mathcal{F}$ where $e_{i j}$ is an oriented edge. We denote $\left\|e_{i j}\right\|=\ell_{i j}$ the edge length. Using this notation, the Finite Element gradient is given by the formula [Botsch et al. 2010]:

$$
\nabla f=\frac{1}{2 \mu\left(T_{i j k}\right)} \mathcal{R}^{90^{\circ}} E_{T_{i j k}}\left(\begin{array}{c}
f_{k}-f_{j} \\
f_{i}-f_{j}
\end{array}\right) .
$$

where $\mathcal{R}^{90^{\circ}}$ denotes the counter-clockwise rotation by $90^{\circ}$. One can remark that the gradient of a function can be expressed in an alternative way depending on the $2 \times 2$-symmetric matrix $\mathbf{g}_{T}$ per triangle:

$$
\nabla f=E_{T} \mathbf{g}_{T}^{-1}\left(\begin{array}{c}
f_{j}-f_{i} \\
f_{k}-f_{j}
\end{array}\right) .
$$

This matrix will be referred to as discrete metric tensor in the local basis $E=\left(e_{i j}, e_{j k}\right)$ of the triangle $T$ :

$$
\mathbf{g}_{T}:=\frac{1}{2}\left(\begin{array}{cc}
2 \ell_{i j}^{2} & \ell_{k i}^{2}-\ell_{j k}^{2}-\ell_{i j}^{2} \\
\ell_{k i}^{2}-\ell_{j k}^{2}-\ell_{i j}^{2} & 2 \ell_{j k}^{2}
\end{array}\right)=E^{\top} E .
$$

Note that $\mathbf{g}_{T}$ is defined such that $\left(\begin{array}{ll}1 & 0\end{array}\right)^{\top} \mathbf{g}_{T}\left(\begin{array}{ll}1 & 0\end{array}\right)=\ell_{i j}^{2}$ so the bilinear form of two adjacent triangle agrees along the edges. Moreover using Heron's formula one can verify that $\operatorname{det}\left(\mathbf{g}_{T}\right)=4 \mu(T)^{2}$.

It follows an alternative expression for the standard cotangent formula:

$$
f^{\top} W g=\sum_{T \in \mathcal{F}}\left(\begin{array}{c}
f_{j}-f_{i} \\
f_{k}-f_{j}
\end{array}\right)^{\top} \mathbf{g}_{T}^{-1}\left(\begin{array}{c}
g_{j}-g_{i} \\
g_{k}-g_{j}
\end{array}\right) \mu(T) .
$$

This formulation is equivalent to the one found in [Boscaini et al. 2015]:

$$
f^{\top} W g=\sum_{T \in \mathcal{F}} \frac{1}{4 \mu(T)}\left(\begin{array}{c}
f_{j}-f_{k} \\
f_{j}-f_{i}
\end{array}\right)^{\top} \mathbf{g}_{T}\left(\begin{array}{c}
g_{j}-g_{k} \\
g_{j}-g_{i}
\end{array}\right),
$$

by noting that any $2 \times 2$ invertible symmetric matrix is linked to its inverse by the formula:

$$
\left(\begin{array}{cc}
0 & -1 \\
1 & 0
\end{array}\right)^{\top} \mathbf{g}_{T}^{-1}\left(\begin{array}{cc}
0 & -1 \\
1 & 0
\end{array}\right)=\frac{1}{4 \mu(T)^{2}} \mathbf{g}_{T}
$$

The classical cotangent weight formula is recovered by noting that $\cot \alpha_{i j}=\left(-\ell_{i j}^{2}+\ell_{j k}^{2}+\ell_{k i}^{2}\right) /(4 \mu(T))$.

\subsection{Discrete Metric Derivative}

First let's remark that the derivative of the discrete metric can be expressed with respect to discrete connection:

LEMMA 2. Given a one parameter family of meshes, the firstorder change in the metric tensor $\mathbf{g}_{T}=E^{\top} E$ and in the area at a triangle $T \in \mathcal{F}$, is given as:

$$
\begin{aligned}
& \left.\frac{\partial_{t} \mathbf{g}_{T}}{\partial t}\right|_{t=0}=E^{\top}\left(\nabla_{E} V\right)_{T}+\left(\nabla_{E} V\right)_{T}^{\top} E, \\
& \left.\frac{\partial_{t} \mu(T)}{\partial t}\right|_{t=0}=\operatorname{div}(u)_{T} \mu(T),
\end{aligned}
$$

where the divergence at triangle $T$ is defined as $\operatorname{div}(u)_{T}:=$ $\operatorname{Tr}\left(\mathbf{g}_{T}^{-1} E^{\top}\left(\nabla_{E} V\right)\right)$.

PROOF. First let's remark that the derivative of the discrete metric can be expressed with respect to discrete connection:

$$
\left.\frac{\partial \mathbf{g}_{T}^{t}}{\partial t}\right|_{t=0}=E^{\top}\left(\nabla_{E} V\right)_{T}+\left(\nabla_{E} V\right)_{T}^{\top} E
$$

Since the metric is linked to the triangle area by $\mu_{t}(T)=$ $\frac{1}{2} \sqrt{\operatorname{det}\left(\mathbf{g}_{T}^{t}\right)}$ the statement obtained by a direct computation of
the derivative. $\quad \square$

ACM Transactions on Graphics, Vol. VV, No. N, Article XXX, Publication date: Month YYYY 


\subsection{Discrete infinitesimal shape difference}

Taking the derivative of the discrete unified shape difference in Eq. (6) might be challenging. However, using the formulation of Eq. (10) leads to an equivalent formulation of Eq. (6) is:

$$
f^{\top} W D_{I}^{t} g:=\sum_{T \in \mathcal{F}}\left\langle\nabla^{t} f, \nabla^{t} g\right\rangle_{T}^{t} \mu(T) .
$$

By taking the derivative of his expression at time $t=0$, we obtain an alternative discretization of the infinitesimal shape difference $E$.

THEOREM 1. The discrete infinitesimal shape difference reads $E^{V}(u)=W_{M}^{-1} H$, where $H$ is a Laplacian matrix whose weights depend on the extrinsic vector field:

$(H)_{i j}=\frac{1}{2} \sum_{j \sim i}\left(c\left(T_{\alpha_{i j}}\right)+c\left(T_{\beta_{i j}}\right)\right)$,

$c(T)=\left(\left\langle e_{j k}, V_{j}-V_{i}\right\rangle+\left\langle e_{i j}, V_{j}-V_{k}\right\rangle\right) \frac{1}{4 \mu(T)}-\operatorname{div}(V)_{T} \frac{\left\langle e_{j k}, e_{k i}\right\rangle}{\mu(T)}$

Proof. Using the FEM gradient, e.g. Eq. (7), to discretize the unified shape difference written in Eq. (12) leads to:

$$
f^{\top} W D_{I}^{t} g=\sum_{T \in \mathcal{F}} \frac{1}{4}\left(\begin{array}{c}
f_{j}-f_{k} \\
f_{j}-f_{i}
\end{array}\right)^{\top} \frac{\mathbf{g}_{T}^{t}}{\mu_{t}(T)^{2}}\left(\begin{array}{c}
g_{j}-g_{k} \\
g_{j}-g_{i}
\end{array}\right) \mu(T) .
$$

We can now compute the derivative with respect to time by using Lemma 2:

$$
\begin{aligned}
& f^{\top} W E^{V} g=\sum_{T \in \mathcal{F}} \frac{1}{4 \mu(T)}\left(\begin{array}{c}
f_{j}-f_{k} \\
f_{j}-f_{i}
\end{array}\right)^{\top} \mathbf{L}_{T}\left(\begin{array}{c}
g_{j}-g_{k} \\
g_{j}-g_{i}
\end{array}\right), \\
& \mathbf{L}_{T}=E^{\top}\left(\nabla_{E} V\right)_{T}+\left(\nabla_{E} V\right)_{T}^{\top} E-2 \operatorname{Tr}\left(\mathbf{g}_{T}^{-1} E^{\top}\left(\nabla_{E} V\right)\right) \mathbf{g}_{T} .
\end{aligned}
$$

The matrices $\mathbf{L}_{T}$ can be written in a form similar to the discrete metric (see Eq. (9)):

$$
\begin{aligned}
& \mathbf{L}_{T}=\frac{1}{2}\left(\begin{array}{cc}
2 a_{i j} & a_{k i}-a_{j k}-a_{i j} \\
a_{k i}-a_{j k}-a_{i j} & 2 a_{j k}
\end{array}\right), \\
& \text { where } a_{i j}=\left\langle e_{i j}, V_{i}-V_{j}\right\rangle-2 \operatorname{div}(V)_{T} \ell_{i j}^{2},
\end{aligned}
$$

where the divergence is define as in Lemma 2 . This leads to the point-wise formulation:

$$
\begin{aligned}
& \left(W_{M} E^{V}\right)_{i j}=\frac{1}{2} \sum_{j \sim i}\left(c\left(T_{\alpha_{i j}}\right)+c\left(T_{\beta_{i j}}\right)\right), \\
& c(T)=\frac{-a_{k i}+a_{j k}+a_{i j}}{4 \mu(T)} .
\end{aligned}
$$

\section{EQUIVALENCE OF THE TWO DISCRETIZATIONS}

PROPOSITION 5. The discretization of $E$ based on the discrete Levi-Civita connection is equivalent to the one obtained by differentiating the unified shape difference operator.

Proof. In Eq. (5) the tangent vectors in a given triangle have be to expressed in the basis form by two edges of the triangle.
Following the discussion in Section 4.2, the FEM gradient at a face $T$ can be written in two equivalent ways:

$$
\nabla f_{T}=\frac{1}{2 \mu(T)} \mathcal{R}^{90^{\circ}} E\left(\begin{array}{c}
f_{j}-f_{k} \\
f_{j}-f_{i}
\end{array}\right)=E \mathbf{g}_{T}^{-1}\left(\begin{array}{c}
f_{j}-f_{i} \\
f_{k}-f_{j}
\end{array}\right) .
$$

Therefore the discrete strain tensor at triangle $T$ in Eq. (5) follows immediately:

$$
\begin{aligned}
\mathcal{L}_{V} \mathbf{g}(\nabla f, \nabla g) & =\left(\begin{array}{c}
f_{i}-f_{j} \\
f_{j}-f_{k}
\end{array}\right)^{\top} \mathbf{g}_{T}^{-1} E^{\top}\left(\bar{\nabla}_{E} V\right) \mathbf{g}_{T}^{-1}\left(\begin{array}{c}
g_{i}-g_{j} \\
g_{j}-g_{k}
\end{array}\right) \\
& +\left(\begin{array}{c}
f_{i}-f_{j} \\
f_{j}-f_{k}
\end{array}\right)^{\top} \mathbf{g}_{T}^{-1}\left(\bar{\nabla}_{E} V\right)^{\top} E \mathbf{g}_{T}^{-1}\left(\begin{array}{c}
g_{i}-g_{j} \\
g_{j}-g_{k}
\end{array}\right) \\
& =\left.\left(\begin{array}{c}
f_{i}-f_{j} \\
f_{j}-f_{k}
\end{array}\right)^{\top} \frac{\partial}{\partial t}\left(\mathbf{g}_{T}^{-1}\right)\right|_{t=0}\left(\begin{array}{c}
g_{i}-g_{j} \\
g_{j}-g_{k}
\end{array}\right) .
\end{aligned}
$$

From Lemma 2, we recognize the term the derivative of the in verse metric. Using Eq. (11), one can further modified the expression to:

$\mathcal{L}_{V} \mathbf{g}(\nabla f, \nabla g)_{T}=-\left.\frac{1}{4}\left(\begin{array}{c}f_{j}-f_{k} \\ f_{j}-f_{i}\end{array}\right)^{\top} \frac{\partial}{\partial t}\left(\frac{\mathbf{g}_{T}^{t}}{\mu_{t}(T)^{2}}\right)\right|_{t=0}\left(\begin{array}{c}g_{j}-g_{k} \\ g_{j}-g_{i}\end{array}\right)$.

The right had side term appears in the discrete isometric shape difference as written in Eq. (13). This leads to the equality between the different discretization:

$$
\left.\frac{\partial}{\partial t}\left(f^{\top} W D_{I}^{t} g\right)\right|_{t=0}=-\sum_{T \in \mathcal{F}} \mathcal{L}_{V} \mathbf{g}(\nabla f, \nabla g)_{T} \mu(T) .
$$

\section{VECTOR FIELDS REPRESENTATION}

PROPOSITION 4. For almost all triangle meshes $M$ without boundary, the operator $E^{V}$ uniquely defines the extrinsic vector field $V$ up to rigid motion.

PROOF. The proof is organized as follow: we show that we can recover the matrices $\mathbf{L}_{T}$ from the infinitesimal shape difference in Eq. (14) then we use a standard results in combinatorix to prove that $\mathbf{L}_{T}=0$ if and only if the extrinsic vector field is a rigid motion.

Kernel of $\mathbf{L}_{T} \mapsto E^{V}$. The information about the extrinsic vector field is solely contained by the matrices $\mathbf{L}_{T}$. Like the discrete metric those matrices agrees across edges so they can be reduced to the vector $a \in \mathbb{R}^{|\mathcal{E}|}$ as defined in Eq. (14). The application $a \mapsto E^{V}$ is linear and we will prove it is almost always invertible.

Extracting elements of $W E^{V}$ corresponding to edges on $M$ yields a linear operator $B: \mathbb{R}^{|\mathcal{E}|} \rightarrow \mathbb{R}^{|\mathcal{E}|}$ with matrix

$$
B_{i j}=\frac{1}{8} \begin{cases}\mu\left(T_{i}\right)^{-1}+\mu\left(T_{i}^{\prime}\right)^{-1} & \text { if } i=j \\ -\mu(T)^{-1} & \text { if } i, j \text { are edges of } T \\ 0 & \text { otherwise. }\end{cases}
$$

Here, indices $i, j$ refer to edges on $M$; for a given edge $i$, we label its adjacent triangles $T_{i}$ and $T_{i}^{\prime}$. Remark that $B$ can be written as a weighted sum: $B=\sum_{k} \frac{1}{8} \mu\left(T_{k}\right)^{-1} B^{k}$, where each $B^{k}$ is a matrix such that:

$$
B_{i j}^{k}=\left\{\begin{array}{l}
1 \quad \text { when } i=j, \text { and } i \text { belongs to triangle } k . \\
-1 \quad \text { when } i, j \text { are edges of triangle } k . \\
0 \quad \text { otherwise. }
\end{array}\right.
$$

It is easy to see that the intersection of the kernels of all $B^{k}$ is empty. Moreover, by considering the determinant of $B$ as a multivariate 


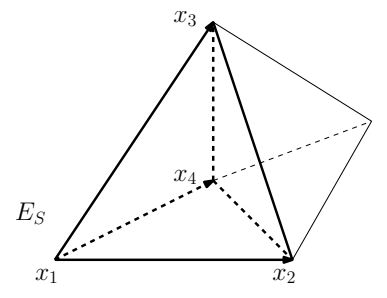

Fig. 1. Local basis in a tetrahedron.

polynomial with real coefficients, we conclude that $B$ is either singular for any choice of values of $\mu\left(T_{k}\right)^{-1}$, or for a finite set of coefficients, which thus have measure zero. The proof of the claim follows by noting that for a closed manifold mesh there must exist a non-singular weighted sum, which can be obtained by iteratively adding triangles, while maintaining that the corresponding matrix sum is non-singular on the incident edges.

Rigidity Theorem. As shown previously the kernel of $a \mapsto E^{V}$ is almost always reduced to the zero element. Going back to the matrices $\mathbf{L}_{T}$, the extrinsic vector field in the kernel should satisfy:

$\mathbf{g}_{T}^{-1} E^{\top}\left(\nabla_{E} V\right)_{T}+\mathbf{g}_{T}^{-1}\left(\nabla_{E} V\right)_{T}^{\top} E-2 \operatorname{Tr}\left(\mathbf{g}_{T}^{-1} E^{\top}\left(\nabla_{E} V\right)\right) \operatorname{Id}=0$.

Taking the trace in both sides implies that $\operatorname{div}(V)_{T}=$ $\operatorname{Tr}\left(\left.\mathbf{g}_{T}^{-1} \partial_{t} \mathbf{g}_{T}^{t}\right|_{0}\right)$ should vanish so it is equivalent to have all matrices $E^{\top}\left(\nabla_{E} V\right)_{T}+\left(\nabla_{E} V\right)_{T}^{\top} E$ equal zero. It follows that the extrinsic vector field satisfies $\left\langle e_{i j}, V_{i}-V_{j}\right\rangle=0$ at all edges. It has been proved in [Gluck 1975] that almost all simply connected closed surfaces only admit rigid deformation as solution of this equation.

\section{CONSTRUCTION FOR TETRAHEDRAL MESHES}

Let's consider the case of a mesh whose constitutive elements are only tetrahedra. The set of tetrahedra is denoted $\mathcal{S}$. Figure 1 illustrates a tetrahedron $S$ defined by 4 vertices $\left(x_{1}, \ldots, x_{4}\right)$. The first 3 edges form the local basis: $E_{S}=\left(x_{2}-x_{1}, \ldots, x_{4}-x_{1}\right)$. The discrete metric tensor, now denoted $\mathrm{g}_{S}=E_{S}^{\top} E_{S}$, is expressed locally using the edge lengths:

$$
\mathbf{g}_{i j}=\left\{\begin{array}{ll}
\ell_{1 i}^{2}, & i=j \\
\frac{1}{2}\left(\ell_{i j}^{2}-\ell_{1 i}^{2}-\ell_{1 j}^{2}\right), & i \neq j .
\end{array}, \quad 1 \leq i, j \leq k .\right.
$$

The volume of the simplex is accessible through the determinant of the metric by $\mu(S)=\sqrt{\operatorname{det}\left(\mathbf{g}_{S}\right)} / 6$. The gradient of a piecewide linear function inside the simplex $S$ is now computed, by analogy with Eq. (8), with the formula:

$$
\nabla f=E_{S} \mathbf{g}_{S}^{-1}\left(\begin{array}{c}
f_{2}-f_{1} \\
\cdots \\
f_{4}-f_{1}
\end{array}\right) .
$$

This formula is strictly equivalent to other more classical FEM formulation as it relies only on computing the derivative of a piecewide linear function.

\subsection{Connection for Tetrahedral Meshes}

Now, the connection of the ambient space $\bar{\nabla}_{u} V$ where $u$ is a vector inside a tetrahedron and $V$ is an extrinsic vector field assigning a vector per vertex:

$$
\begin{aligned}
\bar{\nabla}: \mathbb{R}^{3|\mathcal{S}|} \times \mathbb{R}^{3|\mathcal{V}|} & \rightarrow \mathbb{R}^{3|\mathcal{S}|} \\
(u, V) & \mapsto \bar{\nabla}_{u} V
\end{aligned}
$$

We build the connection $\bar{\nabla}$ by analogy with triangle mesh case in Section 3 leading to add an extra vector in the local basis $E_{S}$.

DEFINITION 4. In a given tetrahedron $S \in \mathcal{S}$ the ambient covariant derivative along the edge $e_{i 1}$ is defined by

$$
\left(\bar{\nabla} \frac{e_{i 1}}{\left\|e_{i 1}\right\|} V\right)_{T}=\frac{V_{i}-V_{1}}{\left\|e_{i 1}\right\|} .
$$

Thus the ambient connection in the directions $E_{S}$ can be stored in a matrix

$$
\left(\bar{\nabla}_{E_{S}} V\right)_{T}=\left(V_{2}-V_{1} \ldots V_{4}-V_{1}\right) .
$$

Then, given any tangent vector $x=E_{S} \alpha$, the covariant derivative in its direction can be computed as $\bar{\nabla}_{x} V=\left(\bar{\nabla}_{E_{S}} V\right) \alpha$.

Given the expression above, the discrete Lie derivative of the metric at simplex $S$ follows immediately. Namely for any pair of tangent vectors $x=E \alpha, y=E \beta$ in the simplex $S$, we have:

$$
\mathcal{L}_{V} \mathbf{g}(x, y)_{S}=\left\langle x,\left(\bar{\nabla}_{E_{S}} V\right) \beta\right\rangle+\left\langle\left(\bar{\nabla}_{E_{S}} V\right) \alpha, y\right\rangle .
$$

Considering the local expression of the gradient in Eq. (16), we obtain:

$$
\begin{aligned}
\mathcal{L}_{V} \mathbf{g}(\nabla f, \nabla g)_{S} & =\left(\begin{array}{c}
f_{2}-f_{1} \\
\ldots \\
f_{4}-f_{1}
\end{array}\right)^{\top} \mathbf{g}_{S}^{-1}\left(\bar{\nabla}_{E_{S}} V\right) \mathbf{g}_{S}^{-1}\left(\begin{array}{c}
g_{2}-g_{1} \\
\ldots \\
g_{4}-g_{1}
\end{array}\right) \\
& +\left(\begin{array}{c}
f_{2}-f_{1} \\
\ldots \\
f_{4}-f_{1}
\end{array}\right)^{\top} \mathbf{g}_{S}^{-1}\left(\bar{\nabla}_{E_{S}} V\right)^{\top} \mathbf{g}_{S}^{-1}\left(\begin{array}{c}
g_{2}-g_{1} \\
\ldots \\
g_{4}-g_{1}
\end{array}\right)
\end{aligned}
$$

After integration we obtain the discrete infinitesimal shape difference on a tetrahedral mesh:

$$
f^{\top} W_{M} E^{V} g=-\sum_{S \in \mathcal{S}} \mathcal{L}_{V} \mathbf{g}(\nabla f, \nabla g)_{S} \mu(S) .
$$

\section{FUNCTIONAL MAP INFERENCE}

PROPOSITION 6. Given a pair of surfaces $M, N$ embedded in $3 D$, and a diffeomorphism $\varphi: N \rightarrow M$, let $C$ be the corresponding functional map $\mathcal{F}_{M} \rightarrow \mathcal{F}_{M}$. Then $M$ and $N$ are related by a rigid motion in space if and only if:

$$
\left\|C_{\varphi} \Delta_{M}-\Delta_{N} C_{\varphi}\right\|+\left\|C_{\varphi} E_{M}^{n}-E_{N}^{n} C_{\varphi}\right\|=0,
$$

where $\Delta$ are the LB operators, while $E^{n}$ are functional deformation fields arising from the normal fields.

Proof. Necessary condition. The commutativity of the functional map with the Laplace-Beltrami operators immediately implies that $\varphi$ is an isometry.

The condition $C_{\varphi} E_{M}^{n}=E_{N}^{n} C_{\varphi}$ will provide an equality between the second fundamental form as $\mathcal{L}_{n} \mathbf{g}=-2 \mathbf{h}$. Let $f, g$ be functions on $M$. Taking the inner product of the left hand side with the function $C_{\varphi}(f)$ and using the isometry property, allows to uncover the second fundamental form of $M$ :

$$
\begin{aligned}
\left\langle C_{\varphi}(f), E_{N}^{n} C_{\varphi}(g)\right\rangle_{H_{0}^{1}(N)} & =\left\langle C_{\varphi}(f), C_{\varphi} E_{M}^{n}(g)\right\rangle_{H_{0}^{1}(N)} \\
& =\left\langle f, E_{M}^{n}(g)\right\rangle_{H_{0}^{1}(M)} \\
& =-2 \int_{M} \mathbf{h}_{M}(\nabla f, \nabla g) \mathrm{d} \mu^{M} .
\end{aligned}
$$

ACM Transactions on Graphics, Vol. VV, No. N, Article XXX, Publication date: Month YYYY. 
The right hand side leads to the pullback of the second fundamental form from $N$ to $M$ :

$$
\begin{aligned}
\left\langle C_{\varphi}(f), E_{N}^{n} C_{\varphi}(g)\right\rangle_{H_{0}^{1}(N)} & =-2 \int_{N} \mathbf{h}_{N}\left(\nabla C_{\varphi}(f), \nabla C_{\varphi}(g)\right) \mathrm{d} \mu^{N} \\
& =-2 \int_{M}\left(\left(\varphi^{-1}\right)^{\star} \mathbf{h}_{N}\right)(\nabla f, \nabla g) \mathrm{d}\left(\varphi_{\star} \mu^{N}\right) \\
& =-2 \int_{M}\left(\left(\varphi^{-1}\right)^{\star} \mathbf{h}_{N}\right)(\nabla f, \nabla g) \mathrm{d} \mu^{M} .
\end{aligned}
$$

Thus for all functions $f, g$, we have:

$$
\int_{M} \mathbf{h}_{M}(\nabla f, \nabla g) \mathrm{d} \mu^{M}=\int_{M}\left(\left(\varphi^{-1}\right)^{\star} \mathbf{h}_{N}\right)(\nabla f, \nabla g) \mathrm{d} \mu^{M} .
$$

Therefore, using a result from [Schumacher 2013], it implies $\mathbf{h}^{M}=\left(\varphi^{-1}\right)^{\star} \mathbf{h}^{N}$

The first and second fundamental forms of $N$ and $M$ agree, so as a consequence of the fundamental theorem of surface theory the two manifolds must relate by a rigid motion.

Sufficient condition. If $N, M$ are equal up to a rigid motion then the first and second fundamental forms are equal. It immediately implies that $C_{\varphi} \Delta_{M}=\Delta_{N} C_{\varphi}$. We show the second equality by reusing the computation done for the necessary condition:

$$
\begin{aligned}
\left\langle C_{\varphi}(f), C_{\varphi} E_{M}^{n}(g)\right\rangle_{H_{0}^{1}(N)} & =\left\langle f, E_{M}^{n}(g)\right\rangle_{H_{0}^{1}(M)} \\
& =-2 \int_{M} \mathbf{h}_{M}(\nabla f, \nabla g) \mathrm{d} \mu^{M} \\
& =-2 \int_{M}\left(\left(\varphi^{-1}\right)^{\star} \mathbf{h}_{N}\right)(\nabla f, \nabla g) \mathrm{d} \mu^{M} \\
& =\left\langle C_{\varphi}(f), E_{N}^{n} C_{\varphi}(g)\right\rangle_{H_{0}^{1}(N)} .
\end{aligned}
$$

Thus, we have $C_{\varphi} E_{M}^{n}=E_{N}^{n} C_{\varphi}$.

\section{INTRINSIC SYMMETRIZATION}

The unified shape difference of composition of mapping can be computed from functional maps and shape differences of the independent maps as shown be the following Lemma.

Lemma 5. Assume that $D_{I}^{\varphi}: H_{0}^{1}(M) \rightarrow H_{0}^{1}(M)$ represents the distortion of the metric between the surfaces $M$ and $P$ induced by the diffeomorphism $\varphi: P \rightarrow M$ and $D_{I}^{\phi}: H_{0}^{1}(P) \rightarrow H_{0}^{1}(P)$ the distortion between the surfaces $P$ and $N$ linked through $\phi$ : $N \rightarrow P$. The distortion $D_{I}^{\varphi \circ \phi}: H_{0}^{1}(M) \rightarrow H_{0}^{1}(M)$ associated to $\varphi \circ \phi: N \rightarrow M$ is given by

$$
D_{I}^{\varphi \circ \phi}=D_{I}^{\varphi} \circ C_{\varphi}^{-1} \circ D_{I}^{\phi} \circ C_{\varphi} .
$$

Proof. The proof relies only on Definition 2:

$$
\begin{aligned}
& \int_{P} C_{\varphi}\left(\left\langle\nabla f, \nabla D_{I}^{\varphi \circ \phi}(g)\right\rangle\right) \mathrm{d} \mu \\
& =\int_{P} C_{\phi}^{-1}(\langle\nabla(f \circ \varphi \circ \phi), \nabla(g \circ \varphi \circ \phi)\rangle) \mathrm{d} \mu \\
& =\int_{P}\left\langle\nabla(f \circ \varphi), \nabla D_{I}^{\phi}(g \circ \varphi)\right\rangle \mathrm{d} \mu \\
& =\int_{P} C_{\varphi}\left(\left\langle\nabla f, \nabla D_{I}^{\varphi}\left(D_{I}^{\phi}(g \circ \varphi) \circ \varphi^{-1}\right)\right\rangle\right) \mathrm{d} \mu .
\end{aligned}
$$

This yields the equality $D_{I}^{\varphi \circ \phi}(g)=D_{I}^{\varphi}\left(D_{I}^{\phi}(g \circ \varphi) \circ \varphi^{-1}\right)$ for all $g \in H_{0}^{1}(M)$.

ACM Transactions on Graphics, Vol. VV, No. N, Article XXX, Publication date: Month YYYY.
Lemma 5 is used to compute the defining condition for intrinsic symmetrization. Namely, we are looking for the diffeomorphism $\varphi: M^{\prime} \rightarrow M$ such that the self-map $\psi=\varphi^{-1} \circ \pi \circ \varphi: M^{\prime} \rightarrow M^{\prime}$ is an isometry or equivalently the unified shape difference $D_{I}^{\psi}$, computed with the map $\psi$, should be equal to identity. Using Prop. 5,

$D_{I}^{\psi}$ becomes:

$$
\begin{aligned}
D_{I}^{\psi} & =D_{I}^{\varphi^{-1}} C_{\varphi} D_{I}^{\pi \circ \varphi} C_{\varphi}^{-1} \\
& =D_{I}^{\varphi^{-1}} C_{\varphi} D_{I}^{\pi} C_{\pi}^{-1} D_{I}^{\varphi} C_{\pi} C_{\varphi}^{-1} \\
& =C_{\varphi}\left(D_{I}^{\varphi}\right)^{-1} D_{I}^{\pi} C_{\pi}^{-1} D_{I}^{\varphi} C_{\pi} C_{\varphi}^{-1} .
\end{aligned}
$$

So the condition $D_{I}^{\psi}=I$ is equivalent to:

$$
D_{I}^{\pi} C_{\pi}^{-1} D_{I}^{\varphi} C_{\pi}=D_{I}^{\varphi} .
$$

\section{REFERENCES}

Boscaini, D., Eynard, D., Kourounis, D., And Bronstein, M. M 2015. Shape-from-operator: Recovering shapes from intrinsic operators. In Computer Graphics Forum. Vol. 34. Wiley Online Library, 265-274. Botsch, M., Kobbelt, L., Pauly, M., Alliez, P., And Lévy, B. 2010. Polygon mesh processing. CRC press.

BREZIS, H. 2010. Functional analysis, Sobolev spaces and partial differential equations. Springer.

Do CARmo, M. 2013. Riemannian Geometry. Mathematics: Theory \& Applications. Birkhäuser Boston.

GLUCK, H. 1975. Almost all simply connected closed surfaces are rigid. In Geometric topology. Springer, 225-239.

Schumacher, H. 2013. Conformal maps and p-dirichlet energies. Tech. rep., Citeseer. 